\title{
Quantum Curie-Weiss Magnet Induced by Violation of Cluster Property
}

\author{
Tomo Munehisa \\ Faculty of Engineering, University of Yamanashi, Kofu, Japan \\ Email: munehisa8tomo@gmail.com
}

How to cite this paper: Munehisa, T. (2020) Quantum Curie-Weiss Magnet Induced by Violation of Cluster Property. World Journal of Condensed Matter Physics, 10, 27-52. https://doi.org/10.4236/wjcmp.2020.102003

Received: January 29, 2020

Accepted: March 13, 2020

Published: March 16, 2020

Copyright $\odot 2020$ by author(s) and Scientific Research Publishing Inc. This work is licensed under the Creative Commons Attribution International License (CC BY 4.0).

http://creativecommons.org/licenses/by/4.0/

\begin{abstract}
There are some concepts that are accepted in our daily life but are not trivial in physics. One of them is the cluster property that means there exist no relations between two events which are sufficiently separated. In the works recently published by the author, the extensive and quantitative examination has been made about the violation of cluster property in the correlation function of the spin operator for the quantum spin system. These works have shown that, when we include the symmetry breaking interaction, the effect by the violation is proportional to the inverse of the system size. Therefore this effect is tinny since the system size is quite large. In order to find the effect due to the violation even when the size is large, we propose a new system where additional spins couple with the spin system on the square lattice, where the coupling constant between these systems being assumed to be small. Applying the perturbation theory, we obtain the effective Hamiltonian for the additional system. This Hamiltonian includes Curie-Weiss model that is induced by the violation of the cluster property. Then we find that this effective Hamiltonian has the factor which is the inverse of the system size. Since Curie-Weiss model, which is known to be exactly soluble, has to contain this factor so that the thermodynamical properties are well-defined, the essential factor for the Hamiltonian is determined by the coupling and the strength of the symmetry breaking interaction. Our conclusion is, therefore, that it is possible to observe the effect by the violation of the cluster property at the inverse temperature whose order is given by these parameters.
\end{abstract}

\section{Keywords}

Quantum Spin, Spontaneous Symmetry Breaking, Cluster Property, Curie-Weiss Model

\section{Introduction}

The concept of entanglement strongly contradicts with the classical one about 
locality, which has been extensively studied by many researchers [1] [2] [3] [4] [5]. One reason for this active study is the possibility of applying entanglement to quantum computer [6] [7] and quantum information [8] [9] [10].

Entanglement found in many-body systems is reviewed in [11]. Its observation was discussed in [12]. Entanglement was discussed in terms of the spontaneous symmetry breaking [13] [14] [15] because the correlation must be found even at the long distance where the whole system changes entirely from the classically ordered state to the disordered one. The work [16] discussed quantum communication when the symmetry breaks spontaneously.

When the system is at the critical point, we could suppose that correlation must be found even in the far distance. By this quite long-range correlation we have to consider that the concept of the cluster property [17] or the cluster decomposition [18] is not trivial. The works [19] [20] discussed the relation between the violation of this property and confinement in QCD. Also we find active studies in quantum field theory [21] [22]. While in the macroscopic system, authors in works [23] [24] studied the cluster property in the term of the stability. Recently another type of the cluster property is discussed in [25].

In the previous paper [26] we have investigated the cluster property of spin $1 / 2 \mathrm{XXZ}$ antiferromagnet on the square lattice. For this antiferromagnet, the ground state realizes semi-classical Neel order [27], in other words, spontaneous symmetry breaking (SSB) [17] [28] of U(1) symmetry. This semi-classical order has been confirmed by spin wave theory [29] and the quantum Monte Carlo method [30] [31]. The review article [32] is quite useful in this order in the spin system. Also see [33] for the experimental review.

The essential point in these studies is that SSB requires the quasi-degenerate states between which the expectation value of the local operator is not zero. The energy difference between these quasi-degenerate states decreases as the lattice size increases. Therefore, in order to determine the ground state definitely, we introduced an additional interaction that explicitly breaks the symmetry. Then we showed that the violation of the cluster property occurs in this model. The magnitude of the violation is order of $1 /(\sqrt{g} N)$, where $g$ is the strength of the explicit symmetry breaking interaction and $N$ is the size of the system. We concluded that it is possible to observe this effect, though it is tinny except for the extremely small $g$. As for the Heisenberg model which has SU(2) symmetry, see [34].

In this paper, we propose another approach which enables us to observe the violation even when $g$ is not so small. We consider a new spin system added to the one on the square lattice we studied in [26]. The whole Hamiltonian is $\hat{H}_{s q}+\hat{V}_{g}+\hat{V}_{e x}$. Here $\hat{H}_{s q}$ denotes the Hamiltonian which operates the states on the square lattice and $\hat{V}_{g}$ is the interaction which breaks $\mathrm{U}(1)$ symmetry explicitly. The newly added interaction, $\hat{V}_{e x}$, consists of spin operators both on the additional system and on the square lattice. It contains a parameter $u$ to represent the strength of the interaction.

Applying the perturbation theory with small $u$, we obtain the effective Hamil- 
tonian $\hat{H}_{e f f, e x}$ for the spins in the additional system. We see that it includes $\mathrm{Cu}$ rie-Weiss model. In this model, it is known that the mean field approximation for the thermodynamic properties gives the exact results. We then find that this effective Hamiltonian $\hat{H}_{e f f, e x}$ has the overall factor $u^{2} /(g N)$. Since Curie-Weiss model has to contain the factor $1 / N$ in order that the thermodynamical properties are well-defined, the essential factor for the system is $u^{2} / g$. We conclude, therefore, that one would be able to observe the violation when the inverse temperature $\beta$ is of order of $\left(u^{2} / g\right)^{-1}$.

Contents of this paper are as follows. In Section 2, we describe our model in some detail. The first subsection is devoted to a brief explanation of the spin $1 / 2$ $\mathrm{XXZ}$ antiferromagnet on the square lattice. Also we collect the results related to the Hamiltonian $\hat{H}_{s q, g}$ [26]. In the second subsection, we define $\hat{V}_{e x}$ which describes an extended part of the model. In Section 3, using the perturbation theory, we derive the effective Hamiltonian $\hat{H}_{e f f, e x}$ from $\hat{V}_{e x}$. A general discussion to derive the effective Hamiltonian is given in appendix A and the concrete form of $\hat{H}_{e f f, e x}$ is calculated in appendix B. We show that the effective Hamiltonian contains Curie-Weiss model, whose Hamiltonian is the square of the sum of all spin operators on the extended sites. We also show that this Hamiltonian $\hat{H}_{\text {eff }, \text { ex }}$ contains the ferromagnet with the finite-range interaction induced by Nambu-Goldstone mode.

In Section 4, we calculate the energy and the specific heat of Curie-Weiss model. For this purpose, we use the mean field approximation, which is discussed in appendix $\mathrm{C}$ in detail. It should be noted that this method is absolutely reliable for the model when the system is infinitely large. In order to assure that our results are sufficiently accurate, we numerically calculate the specific heat on finite lattices.

In Section 5, we investigate the thermodynamic properties of the effective Hamiltonian $\hat{H}_{e f f, e x}$. The first subsection is to calculate the energy and the specific heat when the temperature is high. Here we employ the high temperature expansion described in appendix D. We find the effect by Nambu-Goldstone mode only in this region. In the second subsection, we calculate these thermodynamic properties at a low temperature. Here we employ the mean field approximation which is exact for the ferromagnet due to the degenerate states and reasonable for the one due to Nambu-Goldstone mode. The final section is devoted to summary and discussion.

Since many symbols are used in our paper, we list them in Table 1 for convenience.

\section{Our Model}

\subsection{Spin System on the Square Lattice}

We will consider the quantum spin system on the square lattice. On each site $i$ $\left(i=1, \cdots, N_{s q}\right)$ we have the spin operator $\hat{S}_{i}^{\alpha}(\alpha=x, y, z)$. Then we define the Hamiltonian $\hat{H}_{s q}$ by 
Table 1. Symbols used in our paper. The third column denotes the equation number, if any, where the symbol is defined.

\begin{tabular}{|c|c|c|}
\hline Symbol & Meaning & Def. Equation \\
\hline$\hat{H}_{s q}$ & Hamiltonian on the square lattice & $(1)$ \\
\hline$\hat{H}_{s q, g}$ & Hamiltonian on the square lattice with $\hat{V}_{g}$ & $(5)$ \\
\hline$\hat{H}_{s q, g, D S}$ & Hamiltonian for the degenerate states & (7) \\
\hline$\hat{H}_{s q, g, N G}$ & Hamiltonian for Nambu-Goldstone mode & (9) \\
\hline$\hat{H}_{s q, g, e x}$ & Whole Hamiltonian of the extended system with $\hat{V}_{g}$ & $(11)$ \\
\hline$\hat{H}_{e f f, e x}$ & Effective Hamiltonian on the extended system & $(12)$ \\
\hline$\hat{H}_{C W}$ & Hamiltonian of Curie-Weiss model & $(19)$ \\
\hline$\hat{H}_{\text {small }}$ & Hamiltonian on the $16+8$ lattice & \\
\hline$\hat{H}_{e f f, a, \text { small }}$ & Effective Hamiltonian on the $16+8$ lattice & $(31)$ \\
\hline$\hat{V}_{g}$ & Interaction of the explicit symmetry breaking & (4) \\
\hline$\hat{V}_{e x}$ & Additional interaction for the extended system & $(10)$ \\
\hline$E_{0}\left(N_{s q}\right)$ & Ground state energy of $\hat{H}_{s q}$ for the degenerate states & (7) \\
\hline$E_{G l}$ & $l$-th energy of $\hat{H}_{s q, g, D S}$ & $(50)$ \\
\hline$E_{N G}$ & Ground state energy of $\hat{H}_{s q, g, N G}$ & (9) \\
\hline$E_{C W}\left(J_{C W}\right)$ & Energy of Curie-Weiss model & $(20)$ \\
\hline$E_{e x}$ & Energy by the high temperature expansion & $(27)$ \\
\hline$E_{\text {eff }, a, \text { small }}\left(J, J_{z}\right)$ & Energy of $\hat{H}_{e f f, a, \text { small }}$ & $(32)$ \\
\hline$E_{c a l}\left(u, J_{z}\right)$ & Energy calculated on the small lattice & \\
\hline$N_{s q}$ & Size of the square lattice & \\
\hline$N_{e x}$ & Size of the extended system & \\
\hline$N_{C W}$ & Size of Curie-Weiss model & \\
\hline$g$ & Strength of $\hat{V}_{g}$ & \\
\hline$u$ & Strength of $\hat{V}_{e x}$ & \\
\hline$\hat{S}_{i}^{\alpha}$ & Spin operator on the square lattice & \\
\hline$\hat{S}_{a(i)}^{\alpha}$ & Spin operator in the extended system & \\
\hline$\hat{S}_{a}^{\alpha}$ & Spin operator in Curie-Weiss model & \\
\hline$c(\alpha, i)$ & Coefficient in the first order of $u$ in $\hat{H}_{e f f, e x}$ & $(12)$ \\
\hline$c(\alpha, \beta, i, j)$ & Coefficient in the second order of $u$ in $\hat{H}_{e f f, e x}$ & $(12)$ \\
\hline$c_{D S}(\alpha, \beta, i, j)$ & Second order coefficient due to the degenerate states & $(13)$ \\
\hline$c_{N G}(\alpha, \beta, i, j)$ & Second order coefficient due to Nambu-Goldstone mode & $(13)$ \\
\hline
\end{tabular}




\section{Continued}

\begin{tabular}{cc}
$m_{C W}$ & Solution of mean field appx. in Curie-Weiss model \\
$m$ & Solution of mean field appx. in $\hat{H}_{e f f, e x}$ \\
$C_{v, C W}$ & Specific heat in Curie-Weiss model \\
$C_{v, e x}$ & Specific heat in $\hat{H}_{e f f, e x}$ \\
$\Delta C_{v, e x}$ & Gap of specific heat $C_{v, e x}$ at $\beta_{c}$ \\
$\beta_{c}$ & Critical inverse temperature in $\hat{H}_{e f f, e x}$ \\
\hline
\end{tabular}

$$
\hat{H}_{s q} \equiv \sum_{(i, j)}\left[\hat{S}_{i}^{x} \hat{S}_{j}^{x}+\hat{S}_{i}^{y} \hat{S}_{j}^{y}+\lambda \hat{S}_{i}^{z} \hat{S}_{j}^{z}\right]
$$

Here $(i, j)$ denotes the nearest neighbor pair on the square lattice and $\lambda$ is the parameter between 0 and 1 . The eigen state is given by the linear combination of states $\left|\left\{s_{i}\right\}\right\rangle=\left|s_{1}, s_{2}, \cdots, s_{N_{s q}}\right\rangle$, where $\hat{S}_{i}^{z}\left|s_{i}\right\rangle=\left|s_{i}\right\rangle s_{i} \quad\left(s_{i}= \pm 1 / 2\right)$. The vector space of the states $\left|\left\{s_{i}\right\}\right\rangle$ is denoted by $\mathrm{V}_{\mathrm{sq}}$.

For this antiferromagnet, we divide the whole lattice into two kinds of sub-lattices called A sub-lattice and B sub-lattice. In order to define these sub-lattices we introduce a symbol $P_{i}$ using integers $i_{x}$ and $i_{y}$ for the site $i=i_{x}+i_{y} \sqrt{N_{s q}}$.

$$
P_{i} \equiv \bmod \left(i_{x}+i_{y}, 2\right)= \begin{cases}0 & \text { for } i \in \mathrm{A} \text { sub-lattice } \\ 1 & \text { for } i \in \mathrm{B} \text { sub-lattice }\end{cases}
$$

Then we introduce the spin operator on each sub-lattice,

$$
\begin{gathered}
\hat{S}_{U}^{\alpha} \equiv \sum_{i \in \mathrm{U} \text { sub-lattice }} \hat{S}_{i}^{\alpha}=\sum_{i} \frac{1+\epsilon_{\mathrm{U}}(-1)^{P_{i}}}{2} \hat{S}_{i}^{\alpha}, \\
\epsilon_{\mathrm{U}}=\left\{\begin{array}{ll}
+1 & (\mathrm{U}=\mathrm{A}) \\
-1 & (\mathrm{U}=\mathrm{B})
\end{array} .\right.
\end{gathered}
$$

In order to obtain the ground state, we introduce the symmetry breaking interaction $\hat{V}_{g}$,

$$
\hat{V}_{g} \equiv-g \sum_{i}(-1)^{P_{i}} \hat{S}_{i}^{y}=-g\left[\hat{S}_{A}^{y}-\hat{S}_{B}^{y}\right] \quad(0<g \ll 1) .
$$

Then we have the Hamiltonian $\hat{H}_{s q, g}$,

$$
\hat{H}_{s q, g} \equiv \hat{H}_{s q}+\hat{V}_{g} .
$$

It is well known that in this system there exists Nambu-Goldstone mode, which can be described successfully by spin wave theory. On the other hand, adding the explicit symmetry breaking interaction into the Hamiltonian, we have obtained the lowest energy eigen state and the excited states which are linear combinations of the degenerate states [26] [34]. This leads us to consider two kinds of excited states, which are states due to degenerate states and those from Nambu-Goldstone mode. In order to describe these excited states we will employ two kinds of Hamiltonian $\hat{H}_{s q, g, D S}$ and $\hat{H}_{s q, g, N G}$. 
Following the previous work [26], we present the Hamiltonian $\hat{H}_{s q, g, D S}$ which describes the excited states by the degenerate states $\left|D_{n}\right\rangle$. They are defined by

$$
\hat{Q}\left|D_{n}\right\rangle=\left|D_{n}\right\rangle n, \hat{Q} \equiv \sum_{i} \hat{S}_{i}^{z}
$$

Here $\hat{Q}$ is the generator of $U(1)$ symmetry and $n$ is an integer. Then we define $\hat{H}_{s q, g, D S}$ as

$$
\hat{H}_{s q, g, D S} \equiv E_{0}\left(N_{s q}\right)+a_{s q} \frac{\hat{Q}^{2}}{N_{s q}}+\hat{V}_{g} .
$$

Here $E_{0}\left(N_{s q}\right)$ denotes the lowest energy with $n=0$ and $a_{s q}$ is the constant which is fixed by $\hat{H}_{s q}$. The eigen state $\left|G_{l}\right\rangle$ of $\hat{H}_{s q, g, D S}$ is given by a linear combination of $\left|D_{n}\right\rangle$,

$$
\left|G_{l}\right\rangle=\sum_{n}\left|D_{n}\right\rangle c_{l}(n)
$$

Detailed expression of $\left|G_{l}\right\rangle$ is found in Appendix B.1.

Next we define $\hat{H}_{s q, g, N G}$, which describes the excited states of Nambu-Goldstone mode, based on the spin wave theory.

$$
\hat{H}_{s q, g, N G} \equiv E_{N G}+\sum_{k} \omega_{k} \hat{\alpha}_{k}^{\dagger} \hat{\alpha}_{k}
$$

Here $\hat{\alpha}_{\boldsymbol{k}}$ is the annihilation operator of Nambu-Goldstone mode with the wave vector $\boldsymbol{k}$, and $E_{N G}$ denotes the ground state energy. The effect due to the symmetry breaking interaction $\hat{V}_{g}$ is included in $\omega_{k}$, which is the energy of Nambu-Goldstone mode. Detailed expression of $\omega_{k}$ is given in Appendix B.2.

\subsection{Extended Spin System}

Let us consider a new system which consists of the spin system on the square lattice and the one on $N_{e x}$ additional sites. The state for the additional sites is represented by $\left|\left\{s_{a}\right\}\right\rangle \quad\left(a=1, \cdots, N_{e x}\right)$ where $s_{a}= \pm 1 / 2$. The vector space $\mathrm{V}_{\mathrm{ex}}$ is spanned by these states. We will consider the spin system on the square lattice and the additional spin system. Whole vector space is $\mathrm{V}_{\mathrm{sq}} \otimes \mathrm{V}_{\mathrm{ex}}$. The extended interaction $\hat{V}_{e x}$ is given by

$$
\hat{V}_{e x} \equiv-u \sum_{\alpha=x, y} \sum_{i \in \mathrm{A}} \hat{S}_{\text {sub-lattice }}^{\alpha} \hat{S}_{a(i)}^{\alpha} \quad(u>0) .
$$

Here $a(i)$ is the additional site fixed by the site $i$ as is shown in Figure 1 . Note that the summation for $i$ runs over A sub-lattice only. The whole Hamiltonian $\hat{H}_{s q, g, e x}$ of the system is then defined by

$$
\hat{H}_{s q, g, e x} \equiv \hat{H}_{s q, g}+\hat{V}_{e x} .
$$

\section{Effective Hamiltonian of the Extended Spin System}

In Appendix A we have derived the effective Hamiltonian using the perturbation theory. We apply it to our model, where $\hat{H}_{0}$ is $\hat{H}_{s q, g}$ (5) and the perturbed interaction is $\hat{V}_{e x}(10)$. 


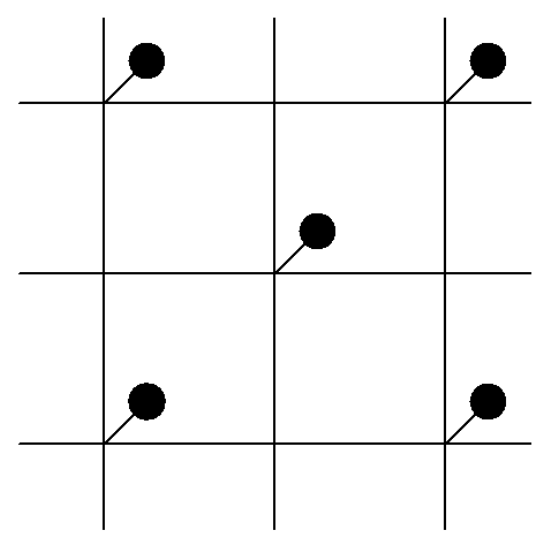

Figure 1. The extended system to the spin system on the square lattice. The ordinal spin is located at the cross point of the horizontal lines and the vertical lines. The line between two nearest points denotes the interaction. The full circle shows the additional spin and the line between the full circle and the cross point shows the additional interaction.

We have two kinds of the excited states. One is the excited state $\left|G_{l}\right\rangle(l \geq 1)$ that consists of the degenerate state and the other is the one-magnon state $|\boldsymbol{k}\rangle$ with the wave vector $\boldsymbol{k}$, which is Nambu-Goldstone mode. Following the discussions in the previous works [26] [34], we suppose that these excited states are independent.

We obtain the effective Hamiltonian $\hat{H}_{\text {eff }, \text { ex }}$ that operates states in $\mathrm{V}_{\mathrm{ex}}$,

$$
\begin{aligned}
\hat{H}_{e f f, e x}= & -u \sum_{\alpha=x, y} \sum_{i \in \mathrm{A} \text { sub-lattice }} c(\alpha, i) \hat{S}_{a(i)}^{\alpha} \\
& +u^{2} \sum_{\alpha, \beta=x, y} \sum_{i, j \in \mathrm{A} \text { sub-lattice }} c(\alpha, \beta, i, j) \hat{S}_{a(i)}^{\alpha} \hat{S}_{a(j)}^{\beta} .
\end{aligned}
$$

Here $c(\alpha, \beta, i, j)$ are sum of the coefficients $c_{D S}(\alpha, \beta, i, j)$ due to the degenerate states and $c_{N G}(\alpha, \beta, i, j)$ due to Nambu-Goldstone mode.

$$
c(\alpha, \beta, i, j)=c_{D S}(\alpha, \beta, i, j)+c_{N G}(\alpha, \beta, i, j) .
$$

From Appendix A we obtain

$$
\begin{gathered}
c(\alpha, i)=\left\langle G_{0}\left|\hat{S}_{i}^{\alpha}\right| G_{0}\right\rangle, \\
c_{D S}(\alpha, \beta, i, j)=\sum_{l \geq 1}\left\langle G_{0}\left|\hat{S}_{i}^{\alpha}\right| G_{l}\right\rangle \frac{1}{E_{G 0}-E_{G l}}\left\langle G_{l}\left|\hat{S}_{j}^{\beta}\right| G_{0}\right\rangle, \\
c_{N G}(\alpha, \beta, i, j)=\sum_{k}\left\langle G_{0}\left|\hat{S}_{i}^{\alpha}\right| \boldsymbol{k}\right\rangle \frac{1}{E_{G 0}-E_{\boldsymbol{k}}}\left\langle\boldsymbol{k}\left|\hat{S}_{j}^{\beta}\right| G_{0}\right\rangle .
\end{gathered}
$$

We have calculated these coefficients in Appendix B. We obtain

$$
c(x, i)=0, c(y, i)=2 v .
$$

Here $v$ denotes the expectation value of the spin operator in the ground state. When we consider the terms of $(\Delta x)^{2}$ only and neglect those of $(\Delta x)^{4}$ for $c_{D S}(\alpha, \beta, i, j)$, we obtain

$$
c_{D S}(x, x, i, j)=2 v^{2} \eta(\Delta x)^{2} \frac{1}{E_{G 0}-E_{G 1}}=-\frac{v}{g N_{s q}},
$$




$$
c_{D S}(x, y, i, j)=0, c_{D S}(y, y, i, j)=0 .
$$

As for contributions by Nambu-Goldstone mode, we obtain

$$
\begin{gathered}
c_{N G}(x, x, i, j) \sim-\frac{1}{4 \pi} K_{0}\left(\tau\left|\boldsymbol{r}_{i}-\boldsymbol{r}_{j}\right|\right), \tau \sim \sqrt{g /(2 v)}, \\
c_{N G}(x, y, i, j)=0, c_{N G}(y, y, i, j)=0 .
\end{gathered}
$$

Here $K_{0}(z)$ is the modified Bessel function. When $N_{s q}$ is as large as $10^{20}$ and $g \gg 1 / \sqrt{N_{s q}}$, the simple expression by the modified Bessel function is reliable for $c_{N G}(x, x, i, j)$ [26]. From (15), (16) and (17) the effective Hamiltonian for our model on the vector space $\mathrm{V}_{\mathrm{ex}}$ is given by

$$
\begin{gathered}
\hat{H}_{e f f, e x}=-2 u v \sum_{i} \hat{S}_{a(i)}^{y}-u^{2} \sum_{i, j \in \mathrm{A} \text { sub-lattice }}\left(-c_{i, j}\right) \hat{S}_{a(i)}^{x} \cdot \hat{S}_{a(j)}^{x}, \\
c_{i, j} \equiv c_{D S}(x, x, i, j)+c_{N G}(x, x, i, j)=-\left\{\frac{v}{g N_{s q}}+\frac{1}{4 \pi} K_{0}\left(\tau\left|\boldsymbol{r}_{i}-\boldsymbol{r}_{j}\right|\right)\right\} .
\end{gathered}
$$

The first term of $c_{i, j}$ has the factor $1 / N_{s q}=1 /\left(2 N_{e x}\right)$ and is independent of the site. We then come to an important conclusion that this effective Hamiltonian contains modified Curie-Weiss model induced by the degenerate state. In the next section, we will discuss this model in some detail.

\section{Curie-Weiss Model}

Curie-Weiss model [35] [36] [37] is defined by, with the site number $N_{C W}$,

$$
\hat{H}_{C W}=-\frac{1}{N_{C W}} \sum_{a=1}^{N_{C W}} \sum_{a^{\prime}=1}^{N_{C W}} \overrightarrow{\hat{S}}_{a} \cdot \overrightarrow{\hat{S}}_{a^{\prime}} .
$$

In this model, we can exactly calculate the specific heat for the infinitely large lattice at any temperature by the mean field approximation. Since this fact is quite important we will make a numerical examination in this section. We compare the specific heat calculated by the eigen values on the large lattices with the result obtained from the mean field approximation.

In Curie-Weiss model, the partition function $Z_{C W}(\beta)$ with the inverse temperature $\beta$ is given by

$$
\begin{gathered}
Z_{C W}(\beta)=\sum_{J_{C W}=0}^{N_{C W} / 2} \operatorname{mul}\left(J_{C W}\right) \mathrm{e}^{-\beta E_{C W}\left(J_{C W}\right)}, \\
E_{C W}\left(J_{C W}\right)=-\frac{J_{C W}\left(J_{C W}+1\right)}{N_{C W}}, \operatorname{mul}\left(J_{C W}\right)=\left(2 J_{C W}+1\right) m u l_{2}\left(J_{C W}\right), \\
\operatorname{mul}_{2}\left(J_{C W}\right)=C\left(N_{C W}, N_{C W} / 2+J_{C W}\right)-C\left(N_{C W}, N_{C W} / 2+J_{C W}+1\right), \\
C(n, k) \equiv \frac{n !}{k !(n-k) !} .
\end{gathered}
$$

Here $N_{C W}$, which we suppose to be even, is the lattice size and $E_{C W}\left(J_{C W}\right)$ denotes the energy eigen value with the magnitude $J_{C W}$ of the total spin.

The multiplicity $\operatorname{mul}\left(J_{C W}\right)$ is given in the following way. First, we consider the 
state of $k$ up-spins and $\left(N_{C W}-k\right)$ down-spins. For this state $M_{C W}$, the $z$-component of the total spin, is given by $M_{C W}=k / 2-\left(N_{C W}-k\right) / 2=k-N_{C W} / 2$. For the fixed $M_{C W}$ we have the multiplicity $m u l_{1}\left(M_{C W}\right)=C\left(N_{C W}, k\right)$ because we pick up $k$ spins among $N_{C W}$ spins. Using $M_{C W}$ instead of $k$ we have $\operatorname{mul}_{1}\left(M_{C W}\right)=C\left(N_{C W}, M_{C W}+N_{C W} / 2\right)$. Since the multiplicity $m u l_{1}\left(J_{C W}\right)$ is a number of possible $J_{C W} \geq M_{C W}$ for each $M_{C W} \geq 0$ the difference $m u l_{1}\left(J_{C W}\right)-m u l_{1}\left(J_{C W}+1\right)$ is the number $\operatorname{mul}_{2}\left(J_{C W}\right)$, which is the multiplicity for the fixed $J_{C W}$. We take account of the multiplicity of $M_{C W}$ for the fixed $J_{C W}$, since the energy $E_{C W}\left(J_{C W}\right)$ does not depend on $M_{C W}$. Thus we obtain the multiplicity of states $\operatorname{mul}\left(J_{C W}\right)$ for the fixed $E_{C W}\left(J_{C W}\right)$.

In Figure 2, we plot the specific heat calculated from the partition function $Z_{C W}(\beta)$ in (20) for $N_{C W}=400,1000,4000$ and 20,000. For comparison we also plot the mean field results we obtain from discussion in Appendix C, noting that $h_{C W}=0$ and $\zeta_{C W}=1$ for Curie-Weiss model (19). When the positive solution $m_{C W}$ of the equation $m_{C W}=\tanh \left(\beta m_{C W}\right) / 2$ exists, the specific heat is given by

$$
C_{v, C W}=\left(\beta m_{C W}\right)^{2} \frac{1-4 m_{C W}^{2}}{1-(\beta / 2)\left(1-4 m_{C W}^{2}\right)} .
$$

Since no positive solution exists for $\beta<\beta_{c}=2$, we have $C_{v, C W}=0$ in this region. Note that this is the characteristic property of Curie-Weiss model. Figure 2 indicates that the result for $N_{C W}=400$ lattice differs from the mean field result, specially around the critical temperature. When the lattice size becomes large, however, the difference clearly decreases. For $N_{C W}=20000$, we find the excellent agreement between the results by both methods except for the narrow region around $\beta_{c}$. We conclude, therefore, the mean field approximation for Curie-Weiss model on large lattices is satisfyingly reliable at any temperature.

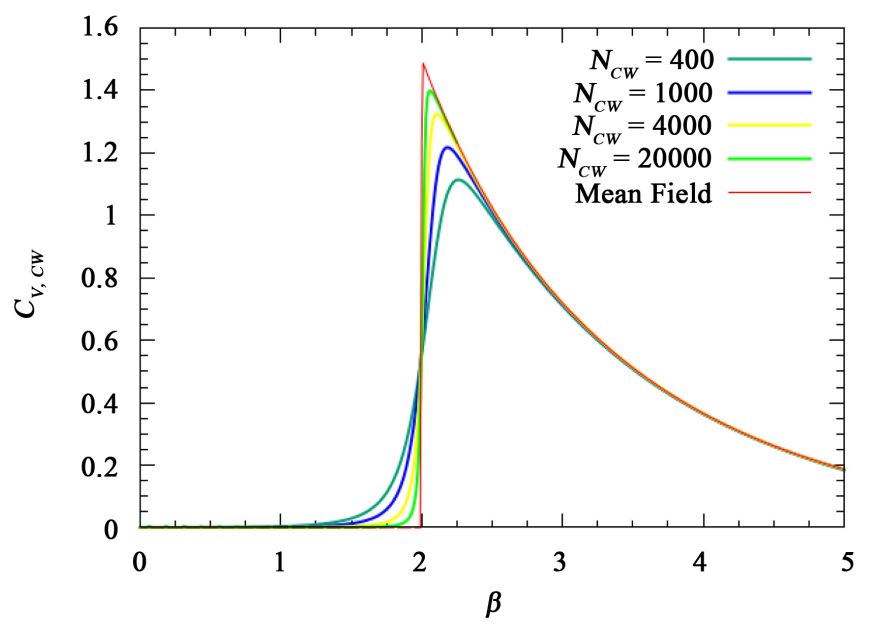

Figure 2. The specific heat of Curie-Weiss model (19) for finite size $N_{C W}$. They are calculated by the exact partition function $Z_{C W}(\beta)$ in (20) for the fixed $N_{C W}$. The red curve is calculated from the mean field approximation, which is given by (21). 


\section{Thermodynamical Properties of the Effective Hamiltonian}

\subsection{High Temperature Region}

Let us study the thermodynamical properties at very small $\beta$. It is known that the high temperature expansion described in Appendix D is a powerful tool in this region. We apply the results by this method to our effective Hamiltonian $\hat{H}_{e f f, e x}$ given in (18), for which

$$
\begin{gathered}
h_{e x}=2 u v, \\
\frac{1}{2} J_{i, j, e x}=u^{2}\left\{c_{D S}(x, x, i, j)+c_{N G}(x, x, i, j)\right\} \sim \frac{u^{2} v}{g N_{s q}}+\frac{u^{2}}{4 \pi} K_{0}\left(\tau\left|\boldsymbol{r}_{i}-\boldsymbol{r}_{j}\right|\right) .
\end{gathered}
$$

Then we have

$$
\begin{aligned}
& \frac{1}{4} \sum_{i}\left(J_{0, i, e x}\right)^{2}=\sum_{i}\left\{\frac{u^{2} v}{g N_{s q}}+\frac{u^{2}}{4 \pi} K_{0}\left(\tau\left|\boldsymbol{r}_{i}\right|\right)\right\}^{2} \\
& =\sum_{i}\left\{\left(\frac{u^{2} v}{g N_{s q}}\right)^{2}+2 \frac{u^{2} v}{g N_{s q}} \frac{u^{2}}{4 \pi} K_{0}\left(\tau\left|\boldsymbol{r}_{i}\right|\right)+\left[\frac{u^{2}}{4 \pi} K_{0}\left(\tau\left|\boldsymbol{r}_{i}\right|\right)\right]^{2}\right\} \\
& =N_{e x}\left(\frac{u^{2} v}{g N_{s q}}\right)^{2}+2 \frac{u^{2} v}{g N_{s q}} \frac{u^{2}}{4 \pi} \sum_{i} K_{0}\left(\tau\left|\boldsymbol{r}_{i}\right|\right)+\frac{u^{4}}{(4 \pi)^{2}} \sum_{i}\left[K_{0}\left(\tau\left|\boldsymbol{r}_{i}\right|\right)\right]^{2} .
\end{aligned}
$$

Here $N_{e x}=N_{s q} / 2$. We obtain, with $x=i_{x} / \Delta, y=i_{y} / \Delta$,

$$
\begin{aligned}
& \sum_{i} K_{0}\left(\tau\left|\boldsymbol{r}_{i}\right|\right)=\sum_{i_{x}, i_{y}} K_{0}\left(\tau \sqrt{i_{x}^{2}+i_{y}^{2}}\right) \rightarrow \frac{1}{\Delta^{2}} \int \mathrm{d} x \int \mathrm{d} y K_{0}\left(\tau \sqrt{x^{2}+y^{2}} / \Delta\right) \\
& =\frac{1}{\Delta^{2}} 2 \pi \int_{0}^{\infty} \mathrm{d} t t K_{0}(\tau t / \Delta)=2 \pi \frac{1}{\Delta^{2}} \frac{\Delta^{2}}{\tau^{2}} \int_{0}^{\infty} \mathrm{d} s s K_{0}(s)=\frac{2 \pi}{\tau^{2}}=\frac{4 \pi v}{g} .
\end{aligned}
$$

Similarly,

$$
\begin{aligned}
& \sum_{i}\left[K_{0}\left(\tau\left|\boldsymbol{r}_{i}\right|\right)\right]^{2} \rightarrow 2 \pi \int_{0}^{\infty} \mathrm{d} r r\left[K_{0}(\tau r)\right]^{2} \\
& =\frac{2 \pi}{\tau^{2}} \int_{0}^{\infty} \mathrm{d} s s\left[K_{0}(s)\right]^{2}=\frac{4 \pi v}{g} K_{2 c}, K_{2 c} \sim 0.5 .
\end{aligned}
$$

Therefore, we obtain

$$
\frac{1}{4} \sum_{i}\left(J_{0, i, e x}\right)^{2} \rightarrow \frac{u^{4}}{4}\left\{\frac{5}{N_{e x}}\left(\frac{v}{g}\right)^{2}+\frac{K_{2 c}}{\pi}\left(\frac{v}{g}\right)\right\} .
$$

Note that the first term of (26) is quite small compared to the second term when $N_{e x}$ is quite large. The energy $E_{e x}$ and the specific heat per site $C_{v, e x}$ are then given by

$$
-\frac{E_{e x}}{N_{e x}} \sim \beta\left[(u v)^{2}+\frac{u^{4} v}{32 \pi g} K_{2 c}\right], C_{v, e x} \sim \beta^{2}\left[(u v)^{2}+\frac{u^{4} v}{32 \pi g} K_{2 c}\right] .
$$

In (27), we see the effect due to the first term of the effective Hamiltonian and that due to Nambu-Goldstone mode only.

\subsection{Low Temperature Region}

In order to calculate the thermodynamical properties at a low temperature, we 
employ the mean field approximation described in Appendix C to the effective Hamiltonian $\hat{H}_{\text {eff }, e x}$. Taking the translational invariance into account and using (24), we obtain

$$
\begin{aligned}
\zeta_{e x} & =\frac{u^{2}}{N_{e x}} \sum_{i, j}\left[\frac{v}{g N_{s q}}+\frac{1}{4 \pi} K_{0}\left(\tau\left|\boldsymbol{r}_{i}-\boldsymbol{r}_{j}\right|\right)\right] \\
& =\frac{u^{2}}{N_{e x}} N_{e x} \sum_{i}\left[\frac{v}{g N_{s q}}+\frac{1}{4 \pi} K_{0}\left(\tau\left|\boldsymbol{r}_{i}\right|\right)\right] \\
\rightarrow & u^{2}\left(\frac{v N_{e x}}{g N_{s q}}+\frac{1}{2 \tau^{2}}\right)=u^{2}\left(\frac{v}{2 g}+\frac{v}{g}\right)=\frac{3 u^{2} v}{2 g} .
\end{aligned}
$$

Let us study how the specific heat $C_{v, e x}$ depends on the parameters $u$ and $g$. Based on results in Appendix $\mathrm{C}$ we see that

$$
C_{v, e x}=\left\{\begin{array}{lc}
\left(\beta \zeta_{e x} m\right)^{2}\left(1-4 m^{2}\right) /\left[1-\left(\beta \zeta_{e x} / 2\right)\left(1-4 m^{2}\right)\right] & \text { for } h_{e x} / \zeta_{e x} \leq 2 m \\
\left(\beta h_{e x} / 2\right)^{2}\left(1-4 m^{2}\right) & \text { otherwise }
\end{array} .\right.
$$

In the case of $h_{e x} / \zeta_{e x} \leq 2 m$, we find the effect due to the degenerate states. Since $m \leq 1 / 2$ we need the condition $h_{e x} / \zeta_{e x} \leq 1$, which is equivalent to $4 g /(3 u) \leq 1$, to observe this effect.

In Figure 3, we plot $C_{v, e x}$ for various values of $4 g /(3 u)$. We find that there exists the gap $\Delta C_{v, e x}$ at the critical temperature $\beta_{c}$. We plot $\beta_{c}$ as a function of $4 g /(3 u)$ in Figure 4, which shows that $\beta_{c}$ is finite when $4 g /(3 u)=0$ while it becomes infinitely large as $4 g /(3 u)$ goes to 1 . Also in Figure 5 , where we show the gap $\Delta C_{v, e x}$, we see that $\Delta C_{v, e x}$ gradually decreases as $4 g /(3 u)$ increases and it vanishes when $4 g /(3 u)$ is 1 .

For the analytic discussion, we expand $\beta_{c}$ and $\Delta C_{v, e x}$ by the polynomial of $h_{e x} / \zeta_{e x}$. At the second order, we obtain

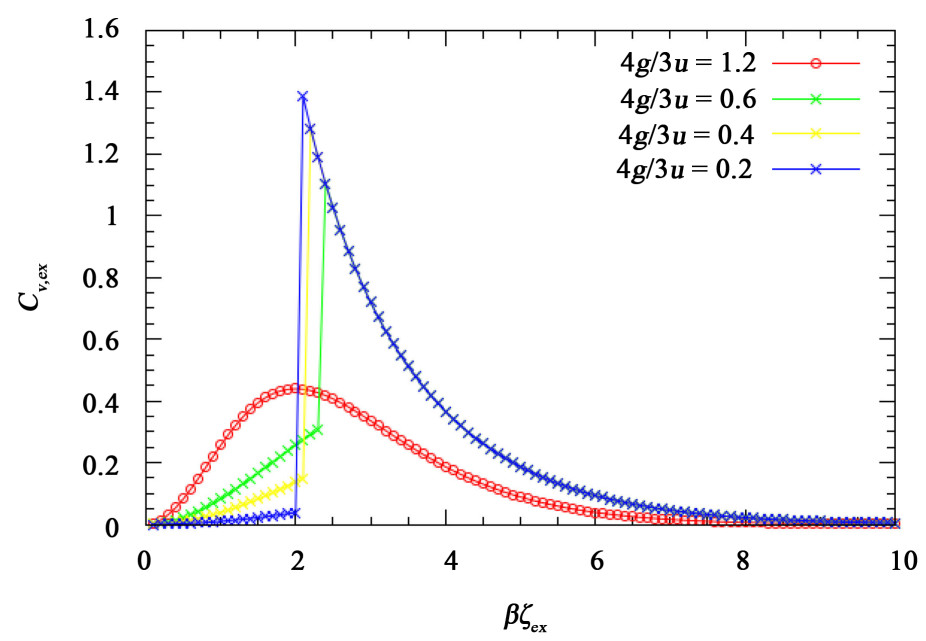

Figure 3. The specific heat of the effective Hamiltonian $\hat{H}_{e f f, e x}$ (18) given by (29) in mean field approximation. The horizontal axis is $\beta \zeta_{e x}$. When $4 g / 3 u \geq 1$ we do not find any gap of the specific heat. If $4 g / 3 u=1$ the gap is $\sim 3 / 2$ as is shown in (30). 


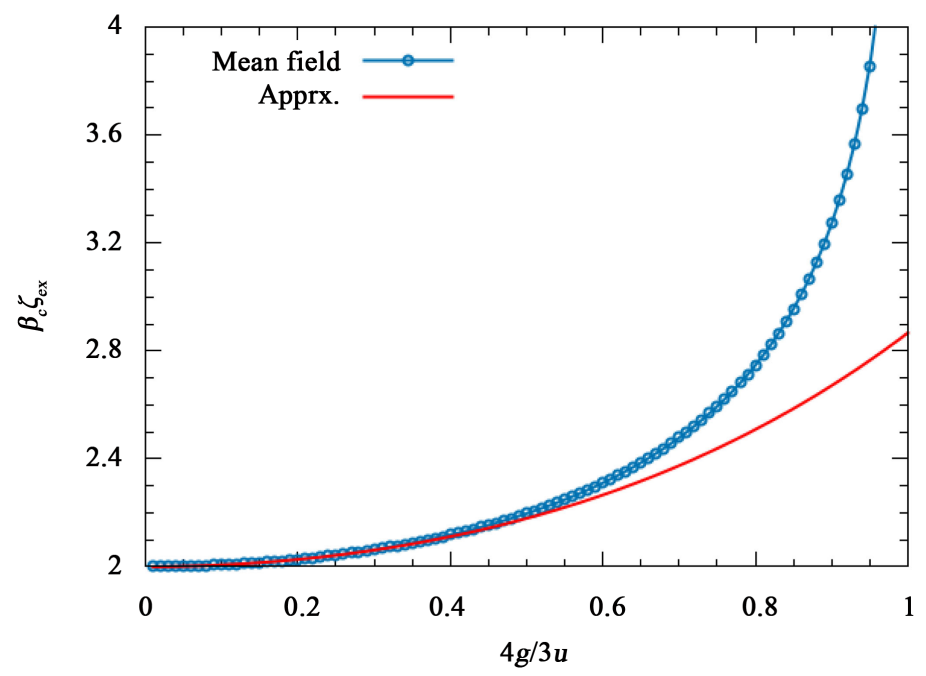

Figure 4. The critical temperature $\beta_{c}$ of the effective Hamiltonian $\hat{H}_{\text {eff ex }}$ in mean field approximation as a function of $4 g /(3 u)$. The vertical axis measures $\beta_{c} \zeta_{\text {ex }}$. The red curve is for the case $g / u \leq 1$, which is given in (30).

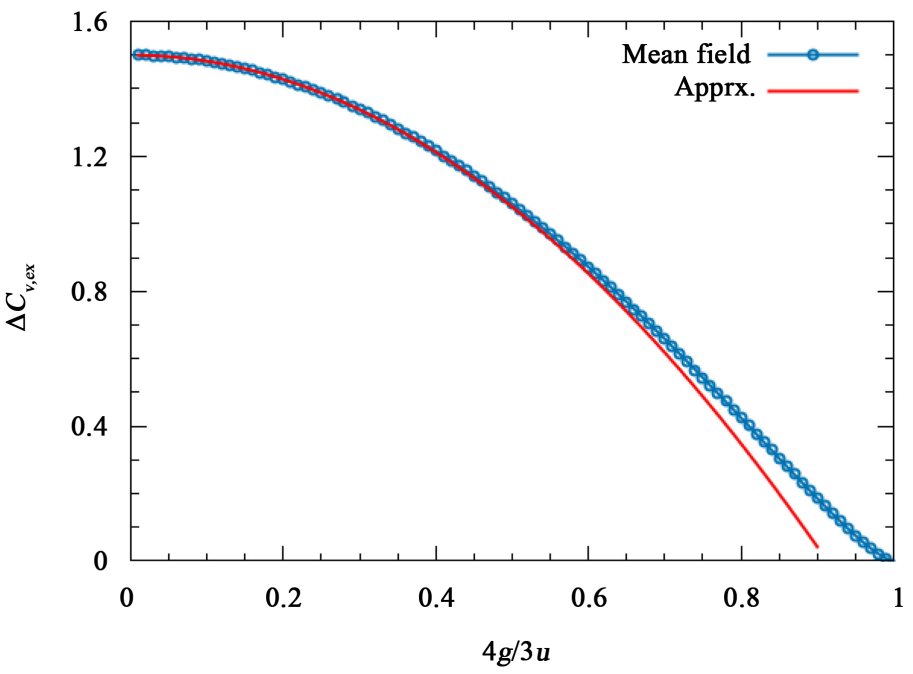

Figure 5. The gap of the specific heat $\Delta C_{v, e x}$ at the critical temperature $\beta_{c}$ in (86) ( $\zeta=\zeta_{e x}$ and $h=h_{e x}$ ) as a function of $h_{e x} / \zeta_{e x}=4 g /(3 u)$. The red curve is for the case $g / u \leq 1$ given in (30).

$$
\begin{aligned}
& \beta_{c} \zeta_{e x} \sim 2\left\{1+\frac{1}{3}\left(\frac{h_{e x}}{\zeta_{e x}}\right)^{2}\right\}=2\left\{1+\frac{1}{3}\left(\frac{4 g}{3 u}\right)^{2}\right\}, \\
& \Delta C_{v, e x} \sim \frac{3}{2}\left\{1-\frac{6}{5}\left(\frac{h_{e x}}{\zeta_{e x}}\right)^{2}\right\}=\frac{3}{2}\left\{1-\frac{6}{5}\left(\frac{4 g}{3 u}\right)^{2}\right\} .
\end{aligned}
$$

These results are also plotted in Figure 4 and Figure 5 for comparison. We see that the polynomial expansion of $h_{e x} / \zeta_{e x}$ is reliable for $h_{e x} / \zeta_{e x}<0.6$. This suggests that the perturbation theory on $h_{e x}$ gives us the good approximation, 
which will be important in future study on the effective Hamiltonian.

Note that if the degenerate states are absent we should use $\zeta_{e x}=u^{2} v / g$ instead of $3 u^{2} v /(2 g)$ because the system is the ferromagnet induced by Nambu-Goldstone mode only. Measuring the specific heat, therefore, we would be able to judge if the degenerate states exist or not.

To summarize this section we present in Figure 6, a region formed by $g$ and $u$, where one can observe the effect by Curie-Weiss model due to the degenerate states. The red curve in the figure gives the boundary for the validity of the perturbation theory. The black curve shows the boundary where we can observe the specific heat by this model. Therefore one can confirm the effect by the violation of the cluster property in the region between the red and the black curves.

\section{Summary and Discussions}

The cluster property is deeply connected with the classical concept about locality, but it is not trivial in quantum physics. In the previous papers [26] [34], we showed the violation of the cluster property (VCP) in spin $1 / 2 \mathrm{XXZ}$ antiferromagnet and Heisenberg antiferromagnet on the square lattice. Our results indicate that the magnitude of VCP is order of $1 /(\sqrt{g} N)$, where $g$ is the strength of the explicit symmetry breaking interaction and $N$ is the size of the system, which we suppose $N \sim 10^{20}$. The observation of VCP in experiments is not easy, therefore, because of its smallness.

In this paper, we proposed an extended spin system so that we find a better chance to observe the effect by VCP. We added a new spin system to the original spin system on the square lattice. The Hamiltonian is $\hat{H}=\hat{H}_{s q, g}+\hat{V}_{e x}$. Here $\hat{H}_{s q, g}$ contains spin operators of the original system only, while $\hat{V}_{e x}$ contains

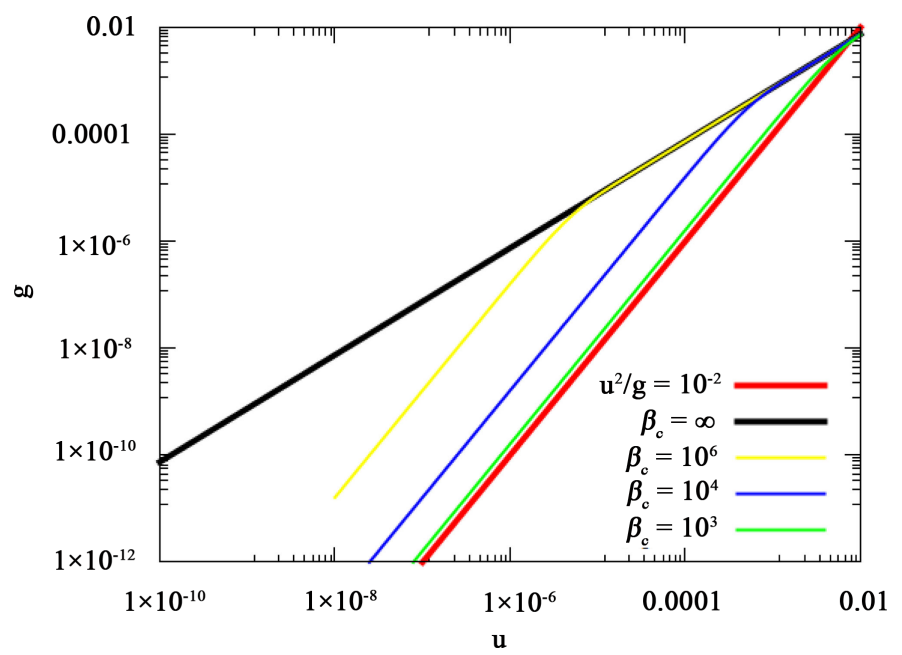

Figure 6. Region of the parameters $g$ and $u$, where we can observe the effect by $\mathrm{Cu}$ rie-Weiss model. For the validity of the perturbation theory we must impose condition $u^{2} / g \leq 10^{-2}$, which is above the red curve. We also need the condition that the critical inverse temperature is finite. The yellow (blue, green) curve shows the values of $g$ and $u$ where the critical inverse temperature $\beta_{c}=10^{6} \quad\left(10^{4}, 10^{2}\right)$. 
spin operators on both systems. Applying the perturbation theory to $\hat{H}$ for a small coupling constant in $\hat{V}_{e x}$, we obtained the effective Hamiltonian $\hat{H}_{\text {eff ex }}$ which operates only on the vector space of the additional system. Then we found that $\hat{H}_{e f f, e x}$ contains Curie-Weiss model induced by the degenerate states. In order to calculate thermodynamic property of the effective Hamiltonian at a low temperature, we employed the mean field approximation, where the difference between the effect due to the degenerate states and that due to Nambu-Goldstone mode is found in the magnitude of the specific heat. Our conclusion is that it is possible to find the effect of the violation of cluster property in our extended model.

Our study in this paper is based on the effective Hamiltonian, which is derived by the perturbation theory. In order to examine the validity of the theory, we consider the Hamiltonian $\hat{H}_{\text {small }} \equiv \hat{H}_{s q}+\hat{V}_{e x}$ on a small lattice, where the energy gap is so large that we do not need the symmetry breaking interaction $\hat{V}_{g}$. Here let us give a brief description of the model and show the results obtained by the diagonalization on the $N_{s q}=16$ and $N_{e x}=8$ lattice $(16+8$ lattice). From (49) in Appendix A the effective Hamiltonian $\hat{H}_{\text {eff }, a \text {,small }}$ reads, $i$ and $j$ being the site on the A sub-lattice,

$$
\hat{H}_{\text {eff }, a, \text { small }}=-\frac{u^{2} v_{\text {small }}^{2}}{\Delta E} \sum_{i, j}\left\{\hat{S}_{a(i)}^{x} \hat{S}_{a(j)}^{x}+\hat{S}_{a(i)}^{y} \hat{S}_{a(j)}^{y}\right\} .
$$

Here we consider only the first excited states so that $\Delta E \equiv E_{1}-E_{0}$, and $v_{\text {small }} \equiv\left\langle 0\left|\hat{S}_{i}^{\alpha}\right| 1\right\rangle \quad(\alpha=x, y)$. Then the energy eigen values for $\hat{H}_{\text {eff } f, a \text {,small }}$ should be given by

$$
E_{\text {eff }, a \text {,small }}\left(J, J_{z}\right)=E_{0}-\frac{u^{2} v_{\text {small }}^{2}}{\Delta E}\left\{J(J+1)-J_{z}^{2}\right\},\left|J_{z}\right| \leq J
$$

For comparison, we directly diagonalize $\hat{H}_{\text {small }}$ on the $16+8$ lattice to obtain the energy eigen values, which we denote $E_{c a l}\left(u, J_{z}\right)$, for fixed values of $u=0.03,0.04$ and $J_{z}=0,1,2,3,4$. By making the least square fitting for $E_{c a l}\left(u, J_{z}\right)$ by $E\left(u, J_{z}\right)=e_{0}+u^{2}\left(e_{1} J_{z}^{2}+e_{2}\right)$, we can estimate the value $0.43(u=0.04)$ and $0.44(u=0.03)$ of $e_{1}$, which should be compared with the value of $v_{\text {small }}^{2} / \Delta E=0.46$ for $\lambda=0.5$. The agreement between both values is satisfactory to assure the validity of the perturbation theory.

Several comments are in order for our calculations and results.

- First let us discuss on effects of higher-order terms in the perturbation theory. On large lattices the energy gap is of order of $\sqrt{g}$ and $\mid\left.\left\langle\right.$ excited state $\left|\hat{V}_{e x}\right|$ ground state $\rangle\right|^{2}$ is of order of $1 /(\sqrt{g} N)$. Then the next order term is of order of $u^{2} /(g N)$, but the factor $N$ should be included into the Hamiltonian for the consistency. Therefore we conclude $u^{2} / g$ should be small in order to neglect higher-order terms.

- Let us consider to estimate the parameters $u$ and $g$ in experiments. One can estimate $u$ by measuring the specific heat at high temperature because, as we have seen in (27), the first term $(u v)^{2}$ dominates compared to the second 
term with the factor $u^{4} v / g$. In order to estimate $g$, on the other hand, one should measure the correlation function of the spin operator which is given in (96) in Appendix D.

- Next we discuss the qualitative difference between the effect due to the degenerate states and that due to Nambu-Goldstone mode. Since we do not see any effect due to the degenerate states at a high temperature, we need to examine the thermodynamic quantities at a low temperature. In this region, where the mean field approximation is valid, it is difficult to distinguish the effect due to the degenerate states from that due to Nambu-Goldstone mode. Therefore we have to investigate the property connected with the excited states which cannot be calculated in the mean field approximation. This subject will be studied in a future work where we investigate the effective Hamiltonian in the extended system with $\mathrm{SU}(2)$ symmetry.

- The last comment is about experimental realization of the proposed spin system. One idea to realize our model is following. In experiments for the spin system on the square lattice, the material contains multi layers. It will be possible to consider the material that has the sandwich structure where the magnetic layer and the quasi non-magnetic layer appear alternately. The magnetic layer realizes the spin system on the square lattice, while in the quasi non-magnetic layer we can partially add the magnetic elements such as $\mathrm{Cu}$. In this additional system, the magnetic elements are sparse so that the coupling between spins on the additional system is weak. Therefore we can suppose that such material realizes $\hat{V}_{e x}$ in our model.

\section{Acknowledgements}

The author specially thanks Dr. Yasuko Munehisa for constructive suggestions and fruitful discussions which are provided through the critical reading of the manuscript.

\section{Conflicts of Interest}

The authors declare no conflicts of interest regarding the publication of this paper.

\section{References}

[1] Bell, J.S. (1964) On the Einstein-Podolsky-Rosen Paradox. Physics, 1, 195. https://doi.org/10.1103/PhysicsPhysiqueFizika.1.195

[2] Kochen, S. and Specker, E.P. (1967) The Problem of Hidden Variables in Quantum Mechanics. Journal of Mathematics and Mechanics, 17, 59-87. https://doi.org/10.1512/iumj.1968.17.17004

[3] Hardy, L. (1993) Nonlocality of Two Particles without Inequalities for Almost All Entangled States. Physics Review Letters, 71, 1665-1668. https://doi.org/10.1103/PhysRevLett.71.1665

[4] 'tHooft, G. (2006) The Mathematical Basis for Deterministic Quantum Mechanics. In: Beyond the Quantum, World Scientific, Singapore, 3-19. 
https://doi.org/10.1142/9789812771186_0001

[5] Horodecki, R., Horodecki, P., Horodecki, M. and Horodecki, K. (2009) Quantum Entanglement. Review of Modern Physics, 81, 865.

https://doi.org/10.1103/RevModPhys.81.865

[6] Deutsch, D. (1985) Quantum Theory, the Church-Turing Principle and the Universal Quantum Computer. Proceedings of the Royal Society of London. Series A, 400, 97. https://doi.org/10.1098/rspa.1985.0070

[7] Nielsen, M.A. and Chuang, I.L. (2000) Quantum Computation and Quantum Information. Cambridge University, Cambridge.

[8] Gühne, O. and Tóth, G. (2009) Entanglement Detection. Physics Reports, 474, 1-75. https://doi.org/10.1016/j.physrep.2009.02.004

[9] Ekert, A., Alves, C., Oi, D., Horodecki, M., Horodecki, P. and Kwek, L. (2002) Direct Estimations of Linear and Nonlinear Functionals of a Quantum State. Physical Review Letters, 88, Article ID: 217901. https://doi.org/10.1103/PhysRevLett.88.217901

[10] Napoli, C., Bromley, T.R., Cianciaruso, M., Piani, M., Johnston, N. and Adesso, G. (2016) Robustness of Coherence: An Operational and Observable Measure of Quantum Coherence. Physical Review Letters, 116, Article ID: 150502. https://doi.org/10.1103/PhysRevLett.116.150502

[11] Amico, L., Fazio, R., Osterloh, A. and Vedral, V. (2008) Entanglement in ManyBody Systems. Review of Modern Physics, 80, 517. https://doi.org/10.1103/RevModPhys.80.517

[12] Islam, R., Ma, R., Preiss, P.M., Tai, M.E., Lukin, A., Rispoli, M. and Greiner, M. (2015) Measuring Entanglement Entropy in a Quantum Many-Body System. Nature, 528, 77. https://doi.org/10.1038/nature15750

[13] Cianciaruso, M., Ferro, L., Giampaolo, S.M., Zonzo, G. and Illuminati, F. (2014) Classical Nature of Ordered Phases: Origin of Spontaneous Symmetry Breaking.

[14] Shi, Y. (2003) Quantum Disentanglement in Long-Range Orders and Spontaneous Symmetry Breaking. Physics Letters A, 309, 254-261. https://doi.org/10.1016/S0375-9601(03)00128-2

[15] Heaney, L., Anders, J., Kaszlikowski, D. and Vedral, V. (2007) Spatial Entanglement from Off-Diagonal Long-Range Order in a Bose-Einstein Condensate. Physics Review $A$, 76, Article ID: 053605. https://doi.org/10.1103/PhysRevA.76.053605

[16] Hamma, A., Giampaolo, S. and Illuminati, F. (2016) Mutual Information and Spontaneous Symmetry Breaking. Physics Review A, 93, Article ID: 012303. https://doi.org/10.1103/PhysRevA.93.012303

[17] Weinberg, S. (1995) The Quantum Theory of Fields. Vol. 2, Cambridge Univ. Press, Cambridge. https://doi.org/10.1017/CBO9781139644167

[18] Strocchi, F. (2008) Symmetry Breaking. Lecture Note Physics 732, Springer, Berlin. https://doi.org/10.1007/978-3-540-73593-9

[19] Strocchi, F. (1978) Local and Covariant Gauge Quantum Field Theories. Cluster Property, Superselection Rules, and the Infrared Problem. Physics Review D, 17, 2010-2021. https://doi.org/10.1103/PhysRevD.17.2010

[20] Lowdon, P. (2016) Conditions on the Violation of the Cluster Decomposition Property in QCD. Journal of Mathematical Physics, 57, Article ID: 102302. https://doi.org/10.1063/1.4965715

[21] Xu, S. and Fan, S. (2017) Generalized Cluster Decomposition Principle Illustrated in Waveguide Quantum Electrodynamics. Physics Review A, 95, Article ID: 063809. https://doi.org/10.1103/PhysRevA.95.063809 
[22] Fröhlich, J. and Rodríguez, P. (2017) On Cluster Properties of Classical Ferromagnets in an External Magnetic Field. Journal of Statistical Physics, 166, 828-840. https://doi.org/10.1007/s10955-016-1556-2

[23] Shimizu, A. and Miyadera, T. (2002) Cluster Property and Robustness of Ground States of Interacting Many Bosons. Journal of the Physical Society of Japan, 71, 56-59. https://doi.org/10.1143/JPSJ.71.56

[24] Shimizu, A. and Miyadera, T. (2002) Stability of Quantum States of Finite Macroscopic Systems against Classical Noises, Perturbations from Environments, and Local Measurements. Physical Review Letters, 89, Article ID: 270403. https://doi.org/10.1103/PhysRevLett.89.270403

[25] Dell'Anna, L., Salberger, O., Barbiero, L., Trombettoni, A. and Korepin, V. (2016) Violation of Cluster Decomposition and Absence of Light Cones in Local Integer and Half-Integer Spin Chains. Physics Review B, 94, Article ID: 155140. https://doi.org/10.1103/PhysRevB.94.155140

[26] Munehisa, T. (2018) Violation of Cluster Property in Quantum Antiferromagnet. World Journal of Condensed Matter Physics, 8, 1-22. https://doi.org/10.4236/wjcmp.2018.81001

[27] Richter, J., Schulenburg, J. and Honecker, A. (2004) Quantum Magnetism. In: Schollwock, U., Richter, J., Farnell, D.J.J. and Bishop, R.F., Eds., Lecture Note in Physics, Volume 645, Springer-Verlag, Berlin Heidelberg, 85-153.

[28] Anderson, P.W. (1984) Basic Notions of Condensed Matter Physics. Benjamin/ Cummings, Menlo Park.

[29] Auerbach, A. (1994) Interacting Electrons and Quantum Magnetism. Springer-Verlag, Berlin Heidelberg. https://doi.org/10.1007/978-1-4612-0869-3

[30] Hatano, N. and Suzuki, M. (1993) Quantum Monte Carlo Methods in Condensed Matter Physics. World Scientific, Singapore, 13-47. https://doi.org/10.1142/9789814503815_0002

[31] De Raedt, H. and von der Linden, W. (1995) The Monte Carlo Method in Condensed Matter Physics. Springer-Verlag, Berlin Heidelberg, 249-284.

[32] Manousakis, E. (1991) The Spin 1/2 Heisenberg Antiferromagnet on a Square Lattice and Its Application to the Cuprous Oxides. Review Modern of Physics, 63, 1. https://doi.org/10.1103/RevModPhys.63.1

[33] Landee, C. and Turnbull, M. (2013) Recent Developments in Low-Dimensional Copper(II) Molecular Magnets. European Journal of Inorganic Chemistry, 2013, 2266-2285. https://doi.org/10.1002/ejic.201300133

[34] Munehisa, T. (2018) Violation of Cluster Property in Heisenberg Antiferromagnet. World Journal of Condensed Matter Physics, 8, 203-229. https://doi.org/10.4236/wjcmp.2018.84015

[35] Kochmanski, M., Paszkiewicz, T. and Wolski, S. (2013) Curie-Weiss Magnet: A Simple Model of Phase Transition. European Journal of Physics, 34.

[36] Chayes, L., Crawford, N., Ioffe, D. and Levit, A. (2008) The Phase Diagram of the Quantum Curie-Weiss Model. Journal of Statistical Physics, 133,131.

[37] Carneiro, C. and Pellegrino, G. (2018) Analysis of Quantum Phase Transition in Some Different Curie-Weiss Models: A Unified Approach.

[38] Predescu, C. (2002) Local Variational Principle. Review Physics Review E, 66, Article ID: 066133. https://doi.org/10.1103/PhysRevE.66.066133

[39] Prato, D. and Barraco, D. (1996) Bogoliubov Inequality. Revista Mexicana de Físiro, 42, 145-150. 


\section{Appendix A}

In this appendix we show how to derive the effective Hamiltonian by means of the perturbation theory. Here we suppose that the whole vector space $\mathrm{V}$ is the direct product of the vector space $\mathrm{V}_{\mathrm{sq}}$ and the vector space $\mathrm{V}_{\mathrm{ex}}$, namely $\mathrm{V}=\mathrm{V}_{\mathrm{sq}} \otimes \mathrm{V}_{\mathrm{ex}}$. We also suppose that the unperturbed Hamiltonian $\hat{H}_{0}$ operates only states on $\mathrm{V}_{\mathrm{sq}}$ and there is no degenerate state for $\hat{H}_{0}$ on the vector space $\mathrm{V}_{\mathrm{sq}}$. They are expressed by

$$
\hat{H}_{0}|l\rangle=|l\rangle E_{l},|l\rangle \in \mathrm{V}_{\mathrm{sq}} .
$$

Here $l \geq 0, E_{l+1}>E_{l}$ and $|0\rangle$ is the lowest energy state. The basis state in $\mathrm{V}_{\mathrm{sq}}$ is given by $|l\rangle$, while the basis state in $\mathrm{V}_{\mathrm{ex}}$ is denoted by $\left|\left\{s_{a}\right\}\right\rangle$. Then the basis state in $\mathrm{V}$ is given by

$$
\left|l,\left\{s_{a}\right\}\right\rangle=|l\rangle\left|\left\{s_{a}\right\}\right\rangle \text {. }
$$

For the unperturbed Hamiltonian $\hat{H}_{0}$,

$$
\hat{H}_{0}\left|l,\left\{s_{a}\right\}\right\rangle=\left|l,\left\{s_{a}\right\}\right\rangle E_{l} .
$$

We suppose that the perturbed Hamiltonian contains the products of the operator $\hat{V}_{s q, o^{\prime}}$ on the vector space $\mathrm{V}_{\mathrm{sq}}$ and the operator $\hat{V}_{e x, o^{\prime}}$ on the vector space $\mathrm{V}_{\mathrm{ex}}$.

$$
\hat{H}=\hat{H}_{0}+u \sum_{o^{\prime}} \hat{V}_{s q, o^{\prime}} \hat{V}_{e x, o^{\prime}}
$$

The eigen state of $\hat{H}$ is given by

$$
|\Phi\rangle=\sum_{\left\{s_{a}\right\}}|0\rangle\left|\left\{s_{a}\right\}\right\rangle c_{0,\left\{s_{a}\right\}}+u \sum_{l \geq 1} \sum_{\left\{s_{a}\right\}}|l\rangle\left|\left\{s_{a}\right\}\right\rangle c_{l,\left\{s_{a}\right\}} .
$$

The coefficient $c_{l,\left\{s_{a}\right\}}$ is a polynomial function of $u$ and contains the term of $u^{0}$. In order to formalize the perturbation theory we employ the variational method, where we introduce a function defined by

$$
F\left(c_{l,\left\{s_{a}\right\}}\right)=\langle\Phi|\hat{H}| \Phi\rangle-\rho\langle\Phi \mid \Phi\rangle .
$$

By the variation on the coefficients we obtain the eigen equation,

$$
\partial F / \partial c_{l,\left\{s_{a}\right\}}=0 .
$$

In order to calculate $F\left(c_{l,\left\{s_{a}\right\}}\right)$ we divide the Hamiltonian $\hat{H}$ to $\hat{H}_{0}$ and the perturbed interactions. For the expectation value of $\hat{H}_{0}$ we obtain, from (37),

$$
\left\langle\Phi\left|\hat{H}_{0}\right| \Phi\right\rangle=\left|c_{0,\left\{s_{a}\right\}}\right|^{2} E_{0}+\sum_{l \geq 1} E_{l}\left|c_{l,\left\{s_{a}\right\}}\right|^{2} u^{2} .
$$

As for the expectation value of $u \hat{V}_{s q, o^{\prime}} \hat{V}_{e x, o^{\prime}}$

$$
\begin{aligned}
& \left\langle\Phi\left|u \hat{V}_{s q, o^{\prime}} \hat{V}_{e x, o^{\prime}}\right| \Phi\right\rangle \\
& =u \sum_{\left\{s_{a}\right\}\left\{s_{a}^{\prime}\right\}}\left\langle 0\left|\hat{V}_{s q, o^{\prime}}\right| 0\right\rangle\left\langle\left\{s_{a}\right\}\left|\hat{V}_{e x, o^{\prime}}\right|\left\{s_{a}^{\prime}\right\}\right\rangle c_{0,\left\{s_{a}^{\prime}\right\}}^{*} c_{0,\left\{s_{a}\right\}} \\
& +u^{2} \sum_{l \geq 1}\left\{\sum_{\left\{s_{a}\right\}\left\{s_{a}^{\prime}\right\}}\left\langle l\left|\hat{V}_{s q, o^{\prime}}\right| 0\right\rangle\left\langle\left\{s_{a}^{\prime}\right\}\left|\hat{V}_{e x, o^{\prime}}\right|\left\{s_{a}\right\}\right\rangle c_{l,\left\{s_{a}^{\prime}\right\}}^{*} c_{0,\left\{s_{a}\right\}}+c . c .\right\} \\
& +u^{3} \sum_{l, l^{\prime} \geq 1}\left\{\sum_{\left\{s_{a}\right\}\left\{s_{a}^{\prime}\right\}}\left\langle l^{\prime}\left|\hat{V}_{s q, o^{\prime}}\right| l\right\rangle\left\langle\left\{s_{a}^{\prime}\right\}\left|\hat{V}_{e x, o^{\prime}}\right|\left\{s_{a}\right\}\right\rangle c_{l^{\prime},\left\{s_{a}^{\prime}\right\}}^{*} c_{l,\left\{s_{a}\right\}}+c . c .\right\} .
\end{aligned}
$$


In the second order perturbation theory, we neglect the terms of $u^{3}$ in $F$. Then the variation on $c_{0,\left\{s_{a}\right\}}$ becomes

$$
\begin{aligned}
\partial F / \partial c_{0,\left\{s_{a}\right\}}= & E_{0} c_{0,\left\{s_{a}\right\}}^{*}+u \sum_{o^{\prime}} \sum_{\left\{s_{a}^{\prime}\right\}}\left\langle 0\left|\hat{V}_{s q, o^{\prime}}\right| 0\right\rangle\left\langle\left\{s_{a}^{\prime}\right\}\left|\hat{V}_{e x, o^{\prime}}\right|\left\{s_{a}\right\}\right\rangle c_{0,\left\{s_{a}\right\}}^{*} \\
& +u^{2} \sum_{o^{\prime}} \sum_{l \geq 1\left\{s_{a}^{\prime}\right\}}\left\langle l\left|\hat{V}_{s q, o^{\prime}}\right| 0\right\rangle\left\langle\left\{s_{a}^{\prime}\right\}\left|\hat{V}_{e x, o^{\prime}}\right|\left\{s_{a}\right\}\right\rangle c_{l,\left\{s_{a}^{\prime}\right\}}^{*}-\rho c_{0,\left\{s_{a}\right\}}^{*} .
\end{aligned}
$$

The variation on $c_{l,\left\{s_{a}\right\}}$ is, on the other hand,

$\partial F / \partial c_{l,\left\{s_{a}\right\}}=u^{2} E_{l} c_{l,\left\{s_{a}\right\}}^{*}+u^{2} \sum_{o^{\prime}} \sum_{\left\{s_{a}^{\prime}\right\}}\left\langle 0\left|\hat{V}_{s q, o^{\prime}}\right| l\right\rangle\left\langle\left\{s_{a}^{\prime}\right\}\left|\hat{V}_{e x, o^{\prime}}\right|\left\{s_{a}\right\}\right\rangle c_{0,\left\{s_{a}^{\prime}\right\}}^{*}-\rho c_{l,\left\{s_{a}\right\}}^{*} u^{2}$.

Requesting $\partial F / \partial c_{l,\left\{s_{a}\right\}}=0$ we obtain the expression for $c_{l,\left\{s_{a}\right\}}^{*}$,

$$
c_{l,\left\{s_{a}\right\}}^{*}=\frac{1}{\rho-E_{l}} \sum_{o^{\prime}} \sum_{\left\{s_{a}^{\prime}\right\}}\left\langle 0\left|\hat{V}_{s q, o^{\prime}}\right| l\right\rangle\left\langle\left\{s_{a}^{\prime}\right\}\left|\hat{V}_{e x, o^{\prime}}\right|\left\{s_{a}\right\}\right\rangle c_{0,\left\{s_{a}^{\prime}\right\}}^{*}
$$

We then replace $c_{l,\left\{s_{a}\right\}}^{*}$ in (42) by the above expression (44). The result is

$$
\begin{aligned}
\partial F / \partial c_{0,\left\{s_{a}\right\}}= & E_{0} c_{0,\left\{s_{a}\right\}}^{*}+u \sum_{o^{\prime}} \sum_{\left\{s_{a}^{\prime}\right\}}\left\langle 0\left|\hat{V}_{s q, o^{\prime}}\right| 0\right\rangle\left\langle\left\{s_{a}^{\prime}\right\}\left|\hat{V}_{e x, o^{\prime}}\right|\left\{s_{a}\right\}\right\rangle c_{0,\left\{s_{a}^{\prime}\right\}}^{*} \\
& +u^{2} \sum_{l \geq 1} \sum_{\left\{s_{a}^{\prime}\right\}}\left\{\sum_{o^{\prime}}\left\langle l\left|\hat{V}_{s q, o^{\prime}}\right| 0\right\rangle\left\langle\left\{s_{a}^{\prime}\right\}\left|\hat{V}_{e x, o^{\prime}}\right|\left\{s_{a}\right\}\right\rangle\right\} \\
& \times \frac{1}{\rho-E_{l}}\left\{\sum_{o^{\prime \prime}} \sum_{\left\{s_{a}^{\prime \prime}\right\}}\left\langle 0\left|\hat{V}_{s q, o^{\prime \prime}}\right| l\right\rangle\left\langle\left\{s_{a}^{\prime \prime}\right\}\left|\hat{V}_{e x, o^{\prime \prime}}\right|\left\{s_{a}^{\prime}\right\}\right\rangle c_{0,\left\{s_{a}^{a}\right\}}^{*}\right\}-\rho c_{0,\left\{s_{a}\right\}}^{*} \\
= & E_{0} c_{0,\left\{s_{a}\right\}}^{*}+u \sum_{o^{\prime}} \sum_{\left\{s_{a}^{\prime}\right\}}\left\langle 0\left|\hat{V}_{s q, o^{\prime}}\right| 0\right\rangle\left\langle\left\{s_{a}^{\prime}\right\}\left|\hat{V}_{e x, o^{\prime}}\right|\left\{s_{a}\right\}\right\rangle c_{0,\left\{s_{a}^{\prime}\right\}}^{*} \\
& +u^{2} \sum_{l \geq 1} \sum_{o^{\prime}, o^{\prime}\left\{s_{a}^{*}\right\}}\left\langle l\left|\hat{V}_{s q, o^{\prime}}\right| 0\right\rangle \frac{1}{\rho-E_{l}}\left\langle 0\left|\hat{V}_{s q, o^{\prime}}\right| l\right\rangle\left\langle\left\{s_{a}^{\prime \prime}\right\}\left|\hat{V}_{e x, o^{\prime \prime}} \hat{V}_{e x, o^{\prime}}\right|\left\{s_{a}\right\}\right\rangle c_{0,\left\{s_{a}^{\prime \prime}\right\}}^{*} \\
& -\rho c_{0,\left\{s_{a}\right\}}^{*} .
\end{aligned}
$$

Here we exchange the order of the summation on $\left\{s_{a}^{\prime}\right\}$ and that of $\left\{s_{a}^{\prime \prime}\right\}$ and use

$$
\sum_{\left\{s_{a}^{\prime}\right\}}\left\langle\left\{s_{a}^{\prime}\right\}\left|\hat{V}_{e x, o^{\prime}}\right|\left\{s_{a}\right\}\right\rangle\left\langle\left\{s_{a}^{\prime \prime}\right\}\left|\hat{V}_{e x, o^{\prime \prime}}\right|\left\{s_{a}^{\prime}\right\}\right\rangle=\left\langle\left\{s_{a}^{\prime \prime}\right\}\left|\hat{V}_{e x, o^{\prime \prime}} \hat{V}_{e x, o^{\prime}}\right|\left\{s_{a}\right\}\right\rangle .
$$

From $\partial F / \partial c_{0,\left\{s_{a}\right\}}=0$ we obtain the equation for $\rho$. Since we can replace $1 /\left(\rho-E_{l}\right)$ by $1 /\left(E_{0}-E_{l}\right)$ in the second order of $u$ we obtain the eigen equation on $\rho$,

$$
\begin{gathered}
\left(\rho-E_{0}\right) c_{0,\left\{s_{a}\right\}}^{*}=\sum_{\left\{s_{a}^{\prime}\right\}} c_{0,\left\{s_{a}^{\prime}\right\}}^{*} M t\left(\left\{s_{a}^{\prime}\right\}:\left\{s_{a}\right\}\right), \\
M t\left(\left\{s_{a}^{\prime}\right\}:\left\{s_{a}\right\}\right) \equiv u \sum_{o^{\prime}}\left\langle 0\left|\hat{V}_{s q, o^{\prime}}\right| 0\right\rangle\left\langle\left\{s_{a}^{\prime}\right\}\left|\hat{V}_{e x, o^{\prime}}\right|\left\{s_{a}\right\}\right\rangle \\
+u^{2} \sum_{o^{\prime}, o^{\prime \prime}} \sum_{\geq 1} \frac{1}{E_{0}-E_{l}}\left\langle 0\left|\hat{V}_{s q, o^{\prime}}\right| l\right\rangle\left\langle l\left|\hat{V}_{s q, o^{\prime}}\right| 0\right\rangle\left\langle\left\{s_{a}^{\prime}\right\}\left|\hat{V}_{e x, o^{\prime \prime}} \hat{V}_{e x, o^{\prime}}\right|\left\{s_{a}\right\}\right\rangle .
\end{gathered}
$$

Using this $M\left(\left\{s_{a}^{\prime}\right\}:\left\{s_{a}\right\}\right)$ we introduce the effective Hamiltonian $\hat{H}_{\text {eff }}$ on $\mathrm{V}_{\mathrm{ex}}$ which should satisfy

$$
\left\langle\left\{s_{a}^{\prime}\right\}\left|\hat{H}_{e f f}\right|\left\{s_{a}\right\}\right\rangle=\operatorname{Mt}\left(\left\{s_{a}^{\prime}\right\}:\left\{s_{a}\right\}\right) .
$$


Since the matrix elements of $\hat{H}_{\text {eff }}$ on $\mathrm{V}_{\text {ex }}$ apply to any state, we can express them by the operators on $V_{e x}$. Finally we obtain the effective Hamiltonian

$$
\hat{H}_{e f f}=u \sum_{o^{\prime}}\left\langle 0\left|\hat{V}_{s q, o^{\prime}}\right| 0\right\rangle \hat{V}_{e x, o^{\prime}}+u^{2} \sum_{o^{\prime}, o^{\prime}} \sum_{\geq 1} \frac{1}{E_{0}-E_{l}}\left\langle 0\left|\hat{V}_{s q, o^{\prime \prime}}\right| l\right\rangle\left\langle l\left|\hat{V}_{s q, o^{\prime}}\right| 0\right\rangle \hat{V}_{e x, o^{\prime}} \hat{V}_{e x, o^{\prime}} .
$$

\section{Appendix B}

Here we calculate the inner product $\left\langle 0\left|\hat{V}_{s q, o^{\prime}}\right| l\right\rangle$ in (49), where $\hat{V}_{s q, o^{\prime}}=(-1)^{P_{i}} \hat{S}_{i}^{\alpha}=\hat{S}_{i}^{\alpha} \quad(\alpha=x, y)$ for the site $i$ on the A sub-lattice.

\section{Part 1}

In this subsection we calculate the contributions due to the degenerate states. In [26] we obtained the eigen state $\left|G_{l}\right\rangle$ of $\hat{H}_{s q, g, D S}$ and the eigen energy $E_{G l}$ $(l=0,1,2, \cdots)$, which are given by (with $f=0$ in (28) and (29) of [26])

$$
\begin{gathered}
\hat{H}_{s q, g, D S}\left|G_{l}\right\rangle=\left|G_{l}\right\rangle E_{G l}, \\
E_{G l}=E_{0}\left(N_{s q}\right)-2 g v N_{s q}+\sqrt{a_{s q} g v}(2 l+1), \\
\left|G_{l}\right\rangle=\sum_{n}\left|D_{n}\right\rangle c_{l}(n)=\sum_{n}\left|D_{n}\right\rangle \Psi_{l}(x=n \Delta x) \sqrt{\Delta x}, \\
\Psi_{l}(x)=N_{l} H_{l}(\sqrt{\eta} x) \mathrm{e}^{-\eta x^{2} / 2}, \eta \equiv \sqrt{\frac{a_{s q}}{g v}} \frac{1}{N_{s q}(\Delta x)^{2}} .
\end{gathered}
$$

Here $H_{l}(u)$ denotes the Hermite polynomial and $N_{l}$ is the normalization factor. Note that we do not need any explicit expression for $\Delta x$, since any physical quantity contains the form of $\eta(\Delta x)^{2}$.

For $(-1)^{P_{i}} \hat{S}_{i}^{y}$ we have [26]

$$
\begin{aligned}
& (-1)^{P_{i}} \hat{S}_{i}^{y}\left|G_{l}\right\rangle=v \sum_{n}\left|D_{n}\right\rangle\left[c_{l}(n+1)+c_{l}(n-1)\right] \\
& =v \sum_{n}\left|D_{n}\right\rangle\left\{2 c_{l}(n)+\left[c_{l}(n+1)+c_{l}(n-1)-2 c_{l}(n)\right]\right\} \\
& \rightarrow v \sum_{n}\left|D_{n}\right\rangle\left\{2 \Psi_{l}(x)+(\Delta x)^{2} \frac{\mathrm{d}^{2} \Psi_{l}}{\mathrm{~d} x^{2}}\right\} \sqrt{\Delta x} \sim v \sum_{n}\left|D_{n}\right\rangle \cdot 2 \Psi_{l}(x) \sqrt{\Delta x} .
\end{aligned}
$$

Then we obtain

$$
\left\langle G_{0}\left|(-1)^{P_{i}} \hat{S}_{i}^{y}\right| G_{l}\right\rangle \rightarrow 2 v \int_{-\infty}^{\infty} \mathrm{d} x \Psi_{0}(x) \Psi_{l}(x)=2 v \delta_{l, 0} .
$$

Similarly we have

$$
\begin{aligned}
& (-1)^{P_{i}} \hat{S}_{i}^{x}\left|G_{l}\right\rangle=-i v \sum_{n}\left|D_{n}\right\rangle\left[c_{l}(n+1)-c_{l}(n-1)\right] \\
& \rightarrow-i v \sum_{n}\left|D_{n}\right\rangle\left[2 \frac{\mathrm{d} \Psi_{l}}{\mathrm{~d} x} \Delta x\right] \sqrt{\Delta x} .
\end{aligned}
$$

Then the inner product is given by

$$
\left\langle G_{0}\left|(-1)^{P_{i}} \hat{S}_{i}^{x}\right| G_{l}\right\rangle \rightarrow-i v \cdot 2 \Delta x \int_{-\infty}^{\infty} \mathrm{d} x \Psi_{0}(x) \frac{\mathrm{d} \Psi_{l}}{\mathrm{~d} x}=-i(\sqrt{\eta} \Delta x) \sqrt{2} v \delta_{l, 1} .
$$

Finally we obtain 


$$
\begin{gathered}
c_{D S}(x, x, i, j)=\left\langle G_{0}\left|\hat{S}_{i}^{x}\right| G_{1}\right\rangle \frac{1}{E_{G 0}-E_{G 1}}\left\langle G_{1}\left|\hat{S}_{j}^{x}\right| G_{0}\right\rangle \\
=2 v^{2} \eta(\Delta x)^{2}\left(\frac{-1}{2 \sqrt{a_{s q} v g}}\right)=-\frac{v}{g N}, \\
c_{D S}(x, y, i, j)=0, c_{D S}(y, y, i, j)=0 .
\end{gathered}
$$

Here we use $\eta(\Delta x)^{2}=\sqrt{a_{s q}} /(\sqrt{g v} N)$ and $E_{G l}=E_{G 0}+2 \sqrt{a_{s q} v g} l$.

\section{Part 2}

Next we discuss the matrix elements due to Nambu-Goldstone mode. We employ the results calculated in the previous work [26] based on spin wave theory. Here the ground state $\left|G_{N G}\right\rangle$ is $\left|G_{0}\right\rangle$ and the excited state is the one magnon state with the wave vector $\boldsymbol{k}$, which we denote by $|\boldsymbol{k}\rangle$.

In spin wave theory, it is known that

$$
\left\langle G_{N G}\left|(-1)^{P_{i}} \hat{S}_{i}^{y}\right| G_{N G}\right\rangle=S,\left\langle\boldsymbol{k}\left|(-1)^{P_{i}} \hat{S}_{i}^{y}\right| G_{N G}\right\rangle=0 .
$$

As for the operator $(-1)^{P_{i}} \hat{S}_{i}^{x}$ we obtain [26]

$$
(-1)^{P_{i}} \hat{S}_{i}^{x}\left|G_{N G}\right\rangle=\frac{\sqrt{2 S}}{2 i \sqrt{N}} \sum_{k}|\boldsymbol{k}\rangle(-1)\left\{\sqrt{\frac{1}{2}\left(1+\frac{1}{\bar{\omega}_{k}}\right)}+\sqrt{\frac{1}{2}\left(-1+\frac{1}{\bar{\omega}_{k}}\right)}\right\} \mathrm{e}^{-i k x_{i}} \text {. }
$$

Here we use the symbols defined by

$$
\begin{gathered}
\omega_{\boldsymbol{k}} \equiv \frac{\omega_{\vec{k}}}{4 S\left(1+\bar{g}+\frac{-1+\lambda}{2} \gamma_{k}\right)}, \\
\omega_{\boldsymbol{k}} \equiv 4 S \sqrt{(1+\bar{g})^{2}+(1+\bar{g})(-1+\lambda) \gamma_{k}-\lambda \gamma_{k}^{2}}, \\
\bar{g} \equiv g /(4 S), \gamma_{k} \equiv\left(\cos k_{x}+\cos k_{y}\right) / 2, \\
\boldsymbol{k}=\left(k_{x}, k_{y}\right) \equiv\left(2 \pi n_{x} / \sqrt{N}, 2 \pi n_{y} / \sqrt{N}\right)\left(n_{x}, n_{y}: \text { integer }\right) .
\end{gathered}
$$

The inner products are then given by

$$
\begin{gathered}
\left\langle G_{N G}\left|(-1)^{P_{i}} \hat{S}_{i}^{x}\right| G_{N G}\right\rangle=0 \\
\left\langle\boldsymbol{k}\left|(-1)^{P_{i}} \hat{S}_{i}^{x}\right| G_{N G}\right\rangle=\frac{\sqrt{2 S}}{2 i \sqrt{N}}(-1)\left\{\sqrt{\frac{1}{2}\left(1+\frac{1}{\bar{\omega}_{\boldsymbol{k}}}\right)}+\sqrt{\frac{1}{2}\left(-1+\frac{1}{\bar{\omega}_{k}}\right)}\right\} \mathrm{e}^{-i k x_{i}} .
\end{gathered}
$$

Therefore we obtain

$$
\begin{gathered}
c_{N G}(x, x, i, j)=\frac{S}{2 N} \sum_{k}\left\{\sqrt{\frac{1}{2}\left(1+\frac{1}{\bar{\omega}_{k}}\right)}+\sqrt{\frac{1}{2}\left(-1+\frac{1}{\bar{\omega}_{k}}\right)}\right\}^{2} \frac{1}{\omega_{k}} \mathrm{e}^{-i k\left(x_{j}-x_{i}\right)}, \\
c_{N G}(y, y, i, j)=0, c_{N G}(x, y, i, j)=0 .
\end{gathered}
$$

For large $r=\left|\boldsymbol{x}_{i}-\boldsymbol{x}_{j}\right|$ the contribution from small $\boldsymbol{k}$ dominates in the sum of $c_{N G}(x, x, i, j)$. For small $|\boldsymbol{k}|$ and small $g$ we see that

$$
\omega_{\boldsymbol{k}} \sim 4 S \sqrt{\frac{1+\lambda}{4}} \sqrt{\tau^{2}+\boldsymbol{k}^{2}}, \bar{\omega}_{\boldsymbol{k}} \sim \frac{1}{\sqrt{1+\lambda}} \sqrt{\tau^{2}+\boldsymbol{k}^{2}},
$$




$$
\tau^{2} \equiv \frac{4 \bar{g}(1+\bar{g}+\lambda)}{1+\lambda+\bar{g}(1-\lambda)} \sim g / S .
$$

Using these approximations and replacing the sum by the integration, we obtain

$$
\begin{aligned}
c_{N G}(x, x, i, j) & \sim \frac{S}{(2 \pi)^{2}} \int \mathrm{d} k_{x} \mathrm{~d} k_{y} \frac{1}{\bar{\omega}_{\boldsymbol{k}} \omega_{\boldsymbol{k}}} \mathrm{e}^{-i k\left(x_{j}-x_{i}\right)} \\
& =\frac{1}{2(2 \pi)^{2}} \int \mathrm{d} k_{x} \mathrm{~d} k_{y} \frac{1}{\tau^{2}+\boldsymbol{k}^{2}} \mathrm{e}^{-i k\left(x_{j}-x_{i}\right)} \\
& =\frac{1}{4 \pi} \int_{0}^{\infty} \mathrm{d} k \frac{k}{\tau^{2}+k^{2}} J_{0}(k r)=\frac{1}{4 \pi} K_{0}(\tau r) .
\end{aligned}
$$

Here we use Bessel functions $J_{0}(z)$ and $K_{0}(z)$.

\section{Appendix C}

The mean field approximation is based on Gibbs-Bogoliubov-Feynman inequality [38] [39], which is given by

$$
\left\langle\mathrm{e}^{W}\right\rangle \geq \mathrm{e}^{\langle W\rangle}
$$

Here $\langle\cdot\rangle$ denotes the statistical average.

Let us apply this inequality to the classical Hamiltonian $H=H_{0}+\left(H-H_{0}\right)$. The statistical average here is defined by

$$
\langle W\rangle_{0}=\left(\sum \mathrm{e}^{-\beta H_{0}} W\right) / \sum \mathrm{e}^{-\beta H_{0}} .
$$

By this definition we have

$$
\sum \mathrm{e}^{-\beta H}=\sum \mathrm{e}^{-\beta H_{0}} \mathrm{e}^{-\beta\left(H-H_{0}\right)}=\left\langle\mathrm{e}^{-\beta\left(H-H_{0}\right)}\right\rangle_{0} \sum \mathrm{e}^{-\beta H_{0}} \geq \mathrm{e}^{-\beta\left\langle H-H_{0}\right\rangle_{0}} \sum \mathrm{e}^{-\beta H_{0}} .
$$

For $Z \equiv \sum \mathrm{e}^{-\beta H}$ and $Z_{0} \equiv \sum \mathrm{e}^{-\beta H_{0}} \quad$ we then obtain

$$
\log Z \geq \log Z_{0}-\beta\left\langle H-H_{0}\right\rangle_{0} .
$$

This inequality is valid for the quantum mechanics, too [38] [39].

$$
\begin{aligned}
& \log Z \geq \log Z_{0}-\beta\left\langle\hat{H}-\hat{H}_{0}\right\rangle_{0}, \\
& \quad Z \equiv \operatorname{tr}\left(\mathrm{e}^{-\beta \hat{H}}\right), Z_{0} \equiv \operatorname{tr}\left(\mathrm{e}^{-\beta \hat{H}_{0}}\right),\langle\hat{A}\rangle_{0} \equiv \operatorname{tr}\left\{\hat{A} \mathrm{e}^{-\beta \hat{H}_{0}}\right\} / \operatorname{tr}\left(\mathrm{e}^{-\beta \hat{H}_{0}}\right) .
\end{aligned}
$$

Let us start our discussion with

$$
\begin{gathered}
\hat{H} \equiv-h \sum_{i=1}^{N} \hat{S}_{i}^{y}-\frac{1}{2} \sum_{i=1}^{N} \sum_{j=1}^{N} J_{i, j} \hat{S}_{i}^{x} \hat{S}_{j}^{x},\left(h \geq 0, J_{i, j} \geq 0, J_{i, i}=0\right), \\
\hat{H}_{0} \equiv-\kappa \sum_{i=1}^{N} \hat{S}_{i}(\kappa>0) .
\end{gathered}
$$

Here we introduce operators,

$$
\hat{S}_{i} \equiv \cos \theta \cdot \hat{S}_{i}^{y}+\sin \theta \cdot \hat{S}_{i}^{x}, \hat{S}_{i}^{\prime} \equiv-\sin \theta \cdot \hat{S}_{i}^{y}+\cos \theta \cdot \hat{S}_{i}^{x} .
$$

The parameters $\kappa$ and $\theta$ are determined later so that they maximize $\log Z_{0}-\beta\left\langle\hat{H}-\hat{H}_{0}\right\rangle_{0}$. It is easy to see that, with $\zeta N \equiv \sum_{i, j} J_{i, j} / 2$,

$$
\log Z_{0}=\log \left\{\operatorname{tr}\left[\mathrm{e}^{\beta \kappa \sum_{i} \hat{S}_{i}}\right]\right\}=N \log [2 \cosh (\beta \kappa / 2)],
$$




$$
\begin{gathered}
\left\langle\hat{S}_{i}\right\rangle_{0}=\frac{1}{2} \tanh (\beta \kappa / 2),\left\langle\hat{S}_{i}^{\prime}\right\rangle_{0}=0, \\
\left\langle\hat{S}_{i}^{y}\right\rangle_{0}=\left\langle\cos \theta \cdot \hat{S}_{i}-\sin \theta \cdot \hat{S}_{i}^{\prime}\right\rangle_{0}=\frac{1}{2} \cos \theta \tanh (\beta \kappa / 2), \\
\left\langle\hat{S}_{i}^{x}\right\rangle_{0}=\left\langle\sin \theta \cdot \hat{S}_{i}+\cos \theta \cdot \hat{S}_{i}^{\prime}\right\rangle_{0}=\frac{1}{2} \sin \theta \tanh (\beta \kappa / 2), \\
\left\langle\hat{H}_{0}\right\rangle_{0}=-N \kappa \frac{1}{2} \tanh (\beta \kappa / 2), \\
\langle\hat{H}\rangle_{0}=-h N \frac{1}{2} \cos \theta \tanh (\beta \kappa / 2)-\zeta N\left[\frac{1}{2} \sin \theta \tanh (\beta \kappa / 2)\right]^{2} .
\end{gathered}
$$

Thus, using $Y \equiv \cos \theta$ and $X \equiv \tanh (\beta \kappa / 2)$ for $\left\langle\hat{H}-\hat{H}_{0}\right\rangle_{0}$, we obtain

$$
\begin{aligned}
& F_{0}(\kappa, Y) \equiv \log Z_{0}-\beta\left\langle\hat{H}-\hat{H}_{0}\right\rangle_{0} \\
& =N\left\{\log [2 \cosh (\beta \kappa / 2)]+\frac{1}{2} \beta h Y X+\left(1-Y^{2}\right) \zeta \frac{1}{4} \beta X^{2}-\beta \kappa \frac{1}{2} X\right\} .
\end{aligned}
$$

In order to find values $\kappa^{*}$ and $Y^{*}$ which maximize $F_{0}(\kappa, Y)$, we examine following equations.

$$
\begin{aligned}
& \frac{\partial}{\partial \kappa}\left\{\frac{1}{N} F_{0}(\kappa, Y)\right\}=\frac{1}{2} \beta\left\{h Y+\left(1-Y^{2}\right) \zeta X-\kappa\right\} \frac{\partial X}{\partial \kappa} \\
&=\frac{1}{2} \beta\{Y(h-\zeta X Y)+\zeta X-\kappa\} \frac{\partial X}{\partial \kappa}=0 . \\
& \frac{\partial}{\partial Y}\left\{\frac{1}{N} F_{0}(\kappa, Y)\right\}=\frac{1}{2} \beta X(h-\zeta X Y)=0 .
\end{aligned}
$$

Since $-1 \leq Y \leq 1$ we see from (73) that, with $X^{*} \equiv \tanh \left(\beta \kappa^{*} / 2\right)$,

$$
Y^{*}=\min \left(\frac{h}{\zeta X^{*}}, 1\right) \text {. }
$$

Let us first consider the case (CASE 1) where $\zeta X^{*}>h$ holds and there exists a positive solution for the equation

$$
\kappa^{*}=\zeta X^{*}=\zeta \tanh \left(\frac{\beta \kappa^{*}}{2}\right) .
$$

Note that $\kappa^{*}>h$ is necessary for this case. The maximum value of $F_{0}(\kappa, Y) / N$ is then

$$
\begin{aligned}
\frac{1}{N} \bar{F}_{0}(\beta) & \equiv \frac{1}{N} F_{0}\left(\kappa^{*}, \frac{h}{\zeta \tanh \left(\beta \kappa^{*} / 2\right)}\right) \\
& =\log \left[2 \cosh \left(\beta \kappa^{*} / 2\right)\right]+\beta \frac{1}{4 \zeta}\left(h^{2}-\kappa^{* 2}\right) .
\end{aligned}
$$

The average energy $E$ and the specific heat per one spin $C_{v}$ are then given by

$$
-\frac{E}{N}=\frac{1}{N} \frac{\mathrm{d} \bar{F}_{0}}{\mathrm{~d} \beta}=\frac{1}{4 \zeta}\left(h^{2}+\kappa^{* 2}\right), C_{v} \equiv-\beta^{2} \frac{\mathrm{d}}{\mathrm{d} \beta} \frac{E}{N}=\frac{\beta^{2} \kappa^{*}}{2 \zeta} \frac{\mathrm{d} \kappa^{*}}{\mathrm{~d} \beta} .
$$

For $\mathrm{d} \kappa^{*} / \mathrm{d} \beta$, we carry out the differential on the condition. 


$$
0=\frac{\mathrm{d}}{\mathrm{d} \beta}\left[\kappa^{*}-\zeta \tanh \left(\beta \kappa^{*} / 2\right)\right]=\frac{\mathrm{d} \kappa^{*}}{\mathrm{~d} \beta}-\zeta \frac{1}{\cosh ^{2}\left(\beta \kappa^{*} / 2\right)}\left[\frac{\kappa^{*}}{2}+\frac{\beta}{2} \frac{\mathrm{d} \kappa^{*}}{\mathrm{~d} \beta}\right] .
$$

Then we obtain

$$
\begin{gathered}
\frac{\mathrm{d} \kappa^{*}}{\mathrm{~d} \beta}=\frac{\zeta \kappa^{*}}{2} \frac{1}{\cosh ^{2}\left(\beta \kappa^{*} / 2\right)-(\beta \zeta / 2)}, \\
C_{v}=\left(\frac{\beta \kappa^{*}}{2}\right)^{2} \frac{1}{\cosh ^{2}\left(\beta \kappa^{*} / 2\right)-(\beta \zeta / 2)} .
\end{gathered}
$$

Next let us consider another case $Y^{*}=1$ (CASE 2), which means $\theta=0$. Note that $h>0$ is necessary for this case. We have

$$
\begin{gathered}
\frac{1}{N} F_{0}(\kappa, 1)=\log [2 \cosh (\beta \kappa / 2)]+\frac{1}{2} \beta h X-\frac{1}{2} \beta \kappa X, \\
\frac{\partial}{\partial \kappa}\left\{\frac{1}{N} F_{0}(\kappa, 1)\right\}=(h-\kappa) \frac{1}{2} \beta \frac{\partial X}{\partial \kappa} .
\end{gathered}
$$

The solution for $\mathrm{d} F_{0} / \mathrm{d} \kappa=0$ is trivial, which is $\kappa^{*}=h$. Then we obtain

$$
\begin{gathered}
\frac{1}{N} F_{0}(h, 1)=\log \left[2 \cosh \left(\frac{\beta h}{2}\right)\right], \\
-\frac{E}{N}=\frac{h}{2} \tanh \left(\frac{\beta h}{2}\right), C_{v}=\left(\frac{\beta h}{2}\right)^{2} \frac{1}{\cosh ^{2}(\beta h / 2)} .
\end{gathered}
$$

For later use, we employ the quantity $m$ instead of $\kappa^{*}$,

$$
m \equiv \frac{1}{2} \tanh \left(\frac{\beta \kappa^{*}}{2}\right) .
$$

From (70), we see that $m$ is $\left\langle\hat{S}_{i}\right\rangle_{0}$ at $\kappa=\kappa^{*}$. Using this $m$ the energy and the specific heat in CASE 1 are given by

$$
-\frac{E}{N}=\frac{h^{2}}{4 \zeta}+\zeta m^{2}, C_{v}=(\beta \zeta m)^{2} \frac{1-4 m^{2}}{1-(\beta \zeta / 2)\left(1-4 m^{2}\right)} .
$$

Here, since $\kappa^{*}=\zeta X^{*}=2 \zeta m, m$ should satisfy the equation

$$
m=\frac{1}{2} \tanh (\beta \zeta m) .
$$

In CASE 2, where $m=\tanh (\beta h / 2) / 2$, we obtain

$$
-\frac{E}{N}=h m, C_{v}=\left(\frac{\beta h}{2}\right)^{2}\left(1-4 m^{2}\right) .
$$

Finally let us examine whether the Equation (74) has a solution which is greater than $h$. We see that there must be a critical value of $\beta, \beta_{c}$, above which we can find the solution. One can easily see that $\beta_{c}=2 / \zeta$ if $h=0$ and $\beta_{c}>2 / \zeta$ if $h>0$. Also we have to pay attention that there is no solution for any $\beta$ if $h>\zeta$. Therefore we obtain $\beta_{c}=\infty$ when $h=\zeta$. It should be noted that the energy in CASE 1 coincides with that in CASE 2 at $\beta_{c}$ while the specific heat does not. Namely, using $\kappa^{*} \rightarrow h$ in the limit $\beta \rightarrow \beta_{c}+0$ and 


$$
\begin{aligned}
h=\zeta \tanh \left(\beta_{c} h / 2\right), & \\
-\frac{1}{N} \Delta E & \equiv-\frac{1}{N}\left\{\lim _{\beta \rightarrow \beta_{c}+0} E(\beta)-\lim _{\beta \rightarrow \beta_{c}-0} E(\beta)\right\} \\
& =\lim _{\beta \rightarrow \beta_{c}+0}\left\{\frac{1}{4 \zeta}\left(h^{2}+\kappa^{*_{2}}\right)\right\}-\lim _{\beta \rightarrow \beta_{c}-0}\left\{\frac{h}{2} \tanh \left(\frac{\beta h}{2}\right)\right\}=0, \\
\Delta C_{v}\left(\beta_{c}\right) & \equiv \lim _{\beta \rightarrow \beta_{c}+0} C_{v}(\beta)-\lim _{\beta \rightarrow \beta_{c}-0} C_{v}(\beta) \\
& =\left(\frac{\beta_{c} h}{2}\right)^{2}\left\{\frac{1}{\cosh ^{2}\left(\beta_{c} h / 2\right)-\beta_{c} \zeta / 2}-\frac{1}{\cosh ^{2}\left(\beta_{c} h / 2\right)}\right\}>0 .
\end{aligned}
$$

Especially, when $h$ is small, we obtain the results

$$
\beta_{c} \zeta \sim 2\left\{1+\frac{1}{3}\left(\frac{h}{\zeta}\right)^{2}\right\}, \Delta C_{v}\left(\beta_{c}\right) \sim \frac{3}{2}\left\{1-\frac{6}{5}\left(\frac{h}{\zeta}\right)^{2}\right\} .
$$

\section{Appendix D}

The Hamiltonian $\hat{H}$ we consider here is given in (68). The partition function $Z(\beta)$ is defined by

$$
Z(\beta)=\operatorname{tr}[\exp (-\beta \hat{H})] .
$$

In the high temperature expansion $Z(\beta)$ is expanded by small $\beta$,

$$
Z(\beta) \sim \operatorname{tr}\left[1-\beta \hat{H}+\frac{\beta^{2}}{2} \hat{H}^{2}\right] .
$$

We employ the following equations

$$
\begin{aligned}
\operatorname{tr}[1]=2^{N}, \operatorname{tr}\left[\hat{S}_{i}^{\alpha}\right]= & 0, \operatorname{tr}\left[\hat{S}_{i}^{\alpha} \hat{S}_{j}^{\alpha^{\prime}}\right]=2^{N} \frac{1}{4} \delta_{i, j} \delta_{\alpha, \alpha^{\prime}}, \operatorname{tr}\left[\hat{S}_{i}^{\alpha} \hat{S}_{i^{\prime}}^{\alpha^{\prime}} \hat{S}_{i^{\prime \prime}}^{\alpha^{\prime \prime}}\right]=0, \\
\operatorname{tr}\left[\hat{S}_{i_{1}}^{\alpha_{1}} \hat{S}_{i_{2}}^{\alpha_{2}} \hat{S}_{i_{3}}^{\alpha_{3}} \hat{S}_{i_{4}}^{\alpha_{4}}\right]= & 2^{N}\left(\frac{1}{4}\right)^{2}\left\{\delta_{i_{1}, i_{2}} \delta_{\alpha_{1}, \alpha_{2}} \delta_{i_{3}, i_{4}} \delta_{\alpha_{3}, \alpha_{4}}+\delta_{i_{1}, i_{3}} \delta_{\alpha_{1}, \alpha_{3}} \delta_{i_{2}, i_{4}} \delta_{\alpha_{2}, \alpha_{4}}\right. \\
& \left.+\delta_{i_{1}, i_{4}} \delta_{\alpha_{1}, \alpha_{4}} \delta_{i_{2}, i_{3}} \delta_{\alpha_{2}, \alpha_{3}}\right\} .
\end{aligned}
$$

In the last equation, we exclude the case where $\alpha_{1}=\alpha_{2}=\alpha_{3}=\alpha_{4}$ and $i_{1}=i_{2}=i_{3}=i_{4}$.

From the Equation (90) we see that the first order term of $\beta$ vanishes.

$$
\operatorname{tr}[\hat{H}]=0 .
$$

In the second order,

$$
\begin{aligned}
\operatorname{tr}\left[\hat{H}^{2}\right] & =h^{2} \sum_{i, j} \operatorname{tr}\left[\hat{S}_{i}^{y} \hat{S}_{j}^{y}\right]+\frac{1}{4} \sum_{i, j, k, l} J_{i, j} J_{k, l} \operatorname{tr}\left[\hat{S}_{i}^{x} \hat{S}_{j}^{x} \hat{S}_{k}^{x} \hat{S}_{l}^{x}\right] \\
& =h^{2} \sum_{i, j} 2^{N} \frac{1}{4} \delta_{i, j}+\frac{1}{4} \sum_{i, j, k, l} J_{i, j} J_{k, l} 2^{N}\left(\frac{1}{4}\right)^{2}\left\{\delta_{i, j} \delta_{k, l}+\delta_{i, k} \delta_{j, l}+\delta_{i, l} \delta_{j, k}\right\} \\
& =2^{N} \frac{1}{4} h^{2} \sum_{i} 1+\frac{1}{4} 2^{N}\left(\frac{1}{4}\right)^{2} \sum_{i, j}\left(J_{i, j} J_{j, j}+J_{i, j} J_{i, j}+J_{i, j} J_{j, i}\right) \\
& =2^{N} \frac{1}{4} h^{2} \sum_{i} 1+\frac{1}{4} 2^{N}\left(\frac{1}{4}\right)^{2} \sum_{i, j} 2\left(J_{i, j}\right)^{2} \\
& =2^{N} N\left\{\frac{1}{4} h^{2}+\frac{1}{4}\left(\frac{1}{4}\right)^{2} \sum_{i} 2\left(J_{0, i}\right)^{2}\right\} .
\end{aligned}
$$


Here we use $J_{i, j}=J_{j, i}$ and $J_{i, i}=0$ as well as the translational invariance. Thus we obtain

$$
Z(\beta) \sim 2^{N}\left\{1+\frac{\beta^{2}}{2} N\left[\frac{1}{4} h^{2}+\frac{1}{32} \sum_{i}\left(J_{0, i}\right)^{2}\right]\right\} .
$$

Therefore the energy and the specific heat are given by

$$
\begin{gathered}
-\frac{E}{N}=\frac{1}{N} \frac{\partial}{\partial \beta} \log (Z(\beta)) \sim \beta\left[\frac{1}{4} h^{2}+\frac{1}{32} \sum_{i}\left(J_{0, i}\right)^{2}\right], \\
C_{v} \sim \beta^{2}\left[\frac{1}{4} h^{2}+\frac{1}{32} \sum_{i}\left(J_{0, i}\right)^{2}\right] .
\end{gathered}
$$

Finally we consider a correlation function of the spin operator $\hat{S}_{a}^{x}$ which will be useful to estimate the coupling constants of the model. It is defined by

$$
F_{C}\left(\beta, r=\left|\boldsymbol{r}_{b}-\boldsymbol{r}_{b^{\prime}}\right|\right) \equiv \frac{\operatorname{tr}\left\{\mathrm{e}^{-\beta \hat{H}} \hat{S}_{b}^{x} \hat{S}_{b^{\prime}}^{x}\right\}}{\operatorname{tr}\left\{\mathrm{e}^{-\beta \hat{H}}\right\}} \quad\left(b \neq b^{\prime}\right)
$$

For small $\beta$, we obtain

$$
F_{C}(\beta, r) \sim \frac{\operatorname{tr}\left\{(-\beta \hat{H}) \hat{S}_{b}^{x} \hat{S}_{b^{\prime}}^{x}\right\}}{\operatorname{tr}\{1\}}=\beta \sum_{i, j} \frac{1}{2} J_{i, j} \frac{\operatorname{tr}\left\{\hat{S}_{i}^{x} \hat{S}_{j}^{x} \hat{S}_{b}^{x} \hat{S}_{b^{\prime}}^{x}\right\}}{\operatorname{tr}\{1\}}=\beta J_{b, b^{\prime}}\left(\frac{1}{4}\right)^{2}
$$

